\title{
Two-dimensional black hole as a topological coset model of $c=1$ string theory
}

\section{Citation}

Mukhi, Sunil, and Cumrun Vafa. 1993. "Two-Dimensional Black Hole as a Topological

Coset Model of $c=1$ String Theory." Nuclear Physics B 407 (3): 667-705. https:// doi.org/10.1016/0550-3213(93)90094-6.

\section{Permanent link}

http://nrs.harvard.edu/urn-3:HUL.InstRepos:41385055

\section{Terms of Use}

This article was downloaded from Harvard University's DASH repository, and is made available under the terms and conditions applicable to Other Posted Material, as set forth at http:// nrs.harvard.edu/urn-3:HUL.InstRepos:dash.current.terms-of-use\#LAA

\section{Share Your Story}

The Harvard community has made this article openly available.

Please share how this access benefits you. Submit a story.

Accessibility 
HUTP-93/A002

TIFR/TH/93-01

\title{
Two Dimensional Black-hole as a Topological Coset Model of $c=1$ String Theory
}

\author{
Sunil Mukhi \\ Tata Institute of Fundamental Research \\ Homi Bhabha Rd \\ Bombay 400 005, India \\ Cumrun Vafa \\ Lyman Laboratory of Physics \\ Harvard University \\ Cambridge, MA 02138, USA
}

We show that a special superconformal coset (with $\hat{c}=3$ ) is equivalent to $c=1$ matter coupled to two dimensional gravity. This identification allows a direct computation of the correlation functions of the $c=1$ non-critical string to all genus, and at nonzero cosmological constant, directly from the continuum approach. The results agree with those of the matrix model. Moreover we connect our coset with a twisted version of a Euclidean two dimensional black hole, in which the ghost and matter systems are mixed.

$1 / 93$ 


\section{Introduction}

Two dimensional quantum field theories coupled to gravity have been studied in great detail in the last few years (see the review article [1]). In particular the conformal theories for which the central charge is less than or equal to 1 coupled to gravity are believed to make sense on arbitrary genus. The case $c=1$ corresponds to the point where the tachyon is just massless, and beyond that we have a tachyonic theory which is not expected to make sense at all genus (i.e., there must be a kind of phase transition).

It is natural therefore to consider the interesting case of $c=1$ string theory. This has been studied in the matrix model formalism beginning with [2] and considerably elaborated in subsequent works (see for example [3] and references therein). There is also a collective field theory description of the target space [4] as well as a Kontsevich-like description (using a generalized Penner model) [5].

Some results have also been obtained from the continuum viewpoint, using the wellknown DDK formalism [6]. This includes the computation of correlators on the sphere [7] and the partition function on the torus [8], and the classification of physical states [9].

Two recent discoveries have uncovered new and interesting aspects of $c=1$ string theory. The first is the discovery of a $2 \mathrm{~d}$ area-preserving symmetry algebra and a natural ring of fields [10] 111] which gives rise to powerful Ward-identities [12]. The second is the observation that the $S L(2, R) / U(1)$ coset, which can be viewed as a two dimensional blackhole, is very similar to the $c=1$ model [13] and this has given rise to the hope that exact computations using the matrix model may shed light on features of two-dimensional black holes (for some recent work in this direction, see [14])

Although some matrix-model results have been re-derived in the continuum DDK formalism, the latter has major limitations, in that it is practicable only in low genus, and one requires certain prescriptions to handle the case of nonzero cosmological constant. It would be highly desirable to have a more powerful continuum formulation in which higher-genus and non-zero cosmological constant are as natural as in matrix models. It has frequently been suggested that a topological field theory could provide the relevant exactly solvable continuum formulation, but so far it has remained an open problem to find the right topological theory.

Two additional results suggest a possible solution to this open problem. The first [15]

is that the $c=1$ string at the self-dual radius computes the Euler characteristic of the moduli space of Riemann surfaces, and can be represented by the double scaling limit of 
the Penner model [16]. The second is the observation [17] that the partition function of a twisted $N=2$ superconformal $S U(2) / U(1)$ coset model at $k=-3$ (with $\hat{c}=3$ ) is also given by the Euler characteristic of the moduli space of Riemann surfaces. This naturally raises the question of whether there is a direct relation between the two. Our primary aim in this paper is to show that they are indeed the same theory. It follows that the coset model provides the desired topological field theory description of the $c=1$ string.

The motivation to reconsider this correspondence came from the recent discovery that there is quite generally an $N=2$ superconformal symmetry in the bosonic string theory [18]. This was further elucidated and generalized [19] to essentially all other known string theories. The simplest description of this $N=2$ symmetry in the bosonic string comes from the observation that the algebra obeyed by the stress-energy tensor, the BRST current, the $b$ ghost and the ghost number current, resembles the twisted $N=2$ superconformal algebra. Indeed, by modifying the BRST and ghost number currents with total derivative terms involving the Liouville field, one can obtain precisely a twisted $N=2$ algebra. Our starting point is that in the $c=1$ string, if instead of the Liouville field one uses the matter field to modify the BRST operator, the resulting theory has $\hat{c}=3$. This suggests a possible correspondence with the superconformal $S U(2) / U(1)$ model at level -3 , which could explain the observations in the previous paragraph.

The organization of this paper is as follows: In section 2 we review how the $N=2$ superconformal symmetry arises in bosonic strings, and how it is realized in the particular case of $c=1$ matter coupled to gravity at zero cosmological constant. In section 3 we discuss the special Kazama-Suzuki (KS) superconformal $S L(2, R) / U(1)$ coset and show that there is a one to one correspondence between physical states of this model and those of the $c=1$ theory in the standard DDK approach. In section 4 we prove that this twisted $\mathrm{KS}$ theory coupled to topological gravity is the same as the $c=1$ theory with non-zero cosmological constant, using the KPZ formalism [20] which turns out to have the same Hilbert space as that of the KS model. In section 5 we show how the computations of [17] for this coset reproduce various results of $c=1$ matrix models.

In section 6 we discuss the black-hole interpretation of this model. The main difference between this description and that of [13] is that here the matter and the ghosts are mixed. We argue why this may be natural and why the KS coset model should be viewed as the correct description of bosonic strings propagating in the 2 dimensional black-hole background. We also make a preliminary study of the implications of this topological symmetry for the black-hole singularity. In section 7 we present our conclusions and some 
further generalizations of these ideas. In this setup it seems that the natural generalization is in terms of twisted $G / G$ theory [21].

In appendix A, which is due to Edward Frenkel, the cohomology of the relevant Kazama-Suzuki model is computed, and is also shown to be equivalent to that of the bosonic $S L(2, R) / S L(2, R)$ topological theory.

\section{2. $c=1$ String Theory as $\mathrm{N}=2$ Topological Field Theory}

It has long been thought that the bosonic string in any background possesses an extended conformal algebra similar to that of the twisted $N=2$ superconformal algebra, where the generators are the total stress-energy tensor $T(z)$ of the matter plus ghost system, the BRST current $G^{+}(z)$, the antighost $b(z)$, and a $U(1)$ current $J(z)$ which counts the ghost number. However, with this choice of generators the algebra does not close, and one has to add two more generators, the ghost $c(z)$ along with $c \partial c(z)$ [22].

More recently, it was understood [18] [19] that with certain modifications, these generators indeed form a twisted $N=2$ algebra which closes without adding any other generators. The modifications consist of adding total derivative terms to the BRST current and the ghost number current. Indeed, if the background has any free scalar field $\eta(z)$ (possibly with a background charge $Q_{\eta}$ ) then the following chiral fields form a twisted $N=2$ superconformal algebra:

$$
\begin{aligned}
T(z) & =T^{M}(z)+T^{G}(z) \\
G^{+}(z) & =c(z) T^{M}(z)+\frac{1}{2}: c(z) T^{G}(z):+x \partial^{2} c(z)+y \partial(c(z) \partial \eta(z)) \\
G^{-}(z) & =b(z) \\
J(z) & =: c b(z):-y \partial \eta(z)
\end{aligned}
$$

where

$$
\begin{aligned}
& x=\frac{1}{2}\left(3+Q_{\eta} y\right) \\
& y=\frac{1}{2}\left(-Q_{\eta}+\sqrt{Q_{\eta}^{2}-8}\right)
\end{aligned}
$$

and the algebra has a topological central charge $\hat{c}=c / 3=2 x$.

In particular, in $c=1$ string theory, one has a Fock space of two free bosons $X(z, \bar{z})$ and $\phi(z, \bar{z})$, the $c=1$ matter field and the $c=25$ Liouville field respectively, and two free fermions, $b(z)$ and $c(z)$, which are the ghost fields. We wish to explore the topological field theory based on the twisted $N=2$ superconformal algebra obtained from this background 
following the above procedure. In principle we have two choices for the scalar field $\eta(z)$ to be used in the algebra. Choosing the Liouville field, however, leads to a difficulty. When we turn on the cosmological constant, the Liouville equation of motion

$$
\partial \bar{\partial} \phi+\mu e^{\phi}=0
$$

means that we cannot consider $\partial \phi$ as a holomorphic current any more. However, in the case of $c=1$ theory we have another option which works even if the cosmological constant is not zero. We choose the $c=1$ matter field $X(z, \bar{z})$ to be $\eta$ in Eq.(2.1) above. Since this field has no background charge, it follows that $x=3 / 2, y=\sqrt{2} i$ and $\hat{c}=3$.

This value of $\hat{c}$ is "critical" in topological field theory [23], and corresponds to the value for Calabi-Yau 3-fold compactifications. Simply put, criticality means that the partition function of such theories can be non-vanishing in every genus, with no need for any insertions. Note that the value of $\hat{c}$ depended only on the background charge $Q_{\eta}$ of the scalar field used in the symmetry algebra. Hence it is a completely general result that "critical" topological theories arise whenever the string theory background has at least one non-anomalous $U(1)$ current.

We now examine how the $N=2$ algebra organizes the states of $c=1$ string theory. To be specific, we will work with this theory at the self-dual radius, where the states are classified by affine $S U(2)$, and restrict our attention in this section to the case where $\mu=0$. In the (chiral) Fock space of $X, \phi, b, c$ we start with the Fock vacua

$$
\left|\Phi_{s, n}^{ \pm}\right\rangle=|\sqrt{2} n\rangle_{X} \otimes|-i \sqrt{2}(1 \mp s)\rangle_{\phi} \otimes c_{1}|0\rangle_{G}
$$

where the subscripts $X, \phi, G$ refer to the matter, Liouville and ghost Fock spaces, and the first two spaces have vacua labelled by momenta, as usual. We have restricted the continuous variable $s$ to be non-negative by explicitly writing its sign, while $n$ runs over half-integers of both signs.

These Fock vacua are created from the $S L(2, C)$ vacuum by the operators

$$
\Phi_{s, n}^{ \pm}(z)=c e^{i \sqrt{2} n X(z)} e^{\sqrt{2}(1 \mp s) \phi(z)}
$$

For each such vacuum and operator, we have a degenerate partner

$$
\left|\Psi_{s, n}^{ \pm}\right\rangle=c_{0}\left|\Phi_{s, n}^{ \pm}\right\rangle
$$


obtained by acting with the $c$-ghost zero mode, and a corresponding field

$$
\Psi_{s, n}^{ \pm}(z)=\partial c(z) \Phi_{s, n}^{ \pm}(z)
$$

For the moment we work only with the holomorphic parts of the fields, although eventually these must be combined with their anti-holomorphic counterparts with the same value and sign of $s$, since the Liouville field is non-compact.

It is easy to check that all these Fock vacua are in fact primaries of the $N=2$ topological algebra. Formally, the operators $\Phi^{ \pm}$look like tachyon vertex operators, but not necessarily on-shell. Their conformal dimension is $n^{2}-s^{2}$. In the particular case when $n= \pm s$, these operators are in the cohomology of $G_{0}^{+}$, and correspond to the physical (on-shell) tachyons of the $c=1$ string.

To find out whether all other Fock-space states are secondaries of the topological algebra, we need to understand the projection map from the $N=2$ module to the Fock space. This map will fail to be bijective if there are null vectors in the former which are in the kernel of the projection. The simplest example shows that this is indeed the case.

Consider the states $\left|\Phi_{1,0}^{+}\right\rangle=c_{1}|0\rangle$ and $\left|\Psi_{1,0}^{+}\right\rangle=c_{0} c_{1}|0\rangle$. Both have conformal dimension $h=-1$, and are mapped onto each other by the action of $G_{0}^{+}$and $G_{0}^{-}$. The $N=2$ algebra generates in principle 8 secondaries at level 1 above these, by the action of $L_{-1}, G_{-1}^{ \pm}$and $J_{-1}$. It is easy to check that four of these are annihilated by all the positive modes of the algebra generators, i.e. they are both primary and secondary, hence null in the $N=2$ module. These are $G_{-1}^{-}\left(c_{1}|0\rangle\right), G_{-1}^{+}\left(c_{1}|0\rangle\right)-\left(L_{-1}-J_{-1}\right)\left(c_{0} c_{1}|0\rangle\right)$, $L_{-1}\left(c_{1}|0\rangle\right)+G_{-1}^{-}\left(c_{0} c_{1}|0\rangle\right)$ and $G_{-1}^{+} c_{0} c_{1}|0\rangle$.

Now we can make the projection to Fock space. The first of the four null states maps to the $S L(2, C)$ Fock vacuum $|0\rangle$, while the other three vanish, so they are in the kernel of the projection. Thus at this level we have one non-vanishing null vector (a Fock space state which is both primary and secondary) and three vanishing null vectors. By general arguments which are well known for the Verma modules of $c=1$ and $c=25$ theories of a single free boson [24] [9], a vanishing null vector gets replaced by a Fock space state which is not a secondary. This can therefore be an extra primary (which is the situation for $c=1$ ) or a state which is neither primary nor secondary (which is the case at $c=25$ ). In the present case we can easily find the extra states, which turn out to be the ones created by the fields $c \partial \phi, c \partial c \partial \phi$ and $c \partial c c \partial^{2} c$. All three are non-primary for the twisted $N=2$ algebra. Thus this situation resembles more closely the case for $c=25$. 
We conclude that the $c=1$ string theory Hilbert space, in the framework of twisted $N=2$ topological symmetry, consists of the Fock vacua in Eqs.(2.4),(2.6), along with $N=2$ secondaries (some of which are null), and extra Fock space states which are neither primary nor secondary (we will argue below that this is true in general).

Let us now continue with the example above and see where the cohomology of the BRST charge $G_{0}^{+}$appears. It is well-known that states in the cohomology appear only for conformal dimension $h=0$. Thus in our example, we may ask which of the five secondaries above $c_{1}|0\rangle$ and $c_{0} c_{1}|0\rangle$, together with the three extra Fock space states, lie in the cohomology. Altogether there are four such states, which are created by the operators $1, c \partial X, c \partial c \partial X$ and $\sqrt{2} \partial c+c \partial \phi$. The first two are in the relative cohomology with respect to $G_{0}^{-}=b_{0}$, and correspond to a Lian-Zuckerman state of ghost number 0 , and a discrete state (the chiral part of the zero-momentum dilaton) of standard ghost number 1 . The other two states are not in the relative cohomology. It is noteworthy that the one state which is a non-vanishing null vector of the $N=2$ algebra is the first Lian-Zuckerman state (of ghost number zero).

One can argue that in fact, all the Lian-Zuckerman states of ghost number 0 are non-vanishing null vectors of the $N=2$ algebra. More precisely, since these states are cohomology classes, the claim is that each class has a representative which is a nonvanishing null vector. To prove this, start with the chiral operators

$$
\begin{aligned}
& x(z)=\left(c b+\frac{i}{\sqrt{2}}(\partial X-i \partial \phi)\right) e^{\frac{i}{\sqrt{2}}(X+i \phi)} \\
& y(z)=\left(c b-\frac{i}{\sqrt{2}}(\partial X+i \partial \phi)\right) e^{-\frac{i}{\sqrt{2}}(X-i \phi)}
\end{aligned}
$$

which generate the ground ring of ghost number zero cohomology classes [10]. One can check explicitly that the commutator of $L_{n}, G_{n}^{ \pm}$and $J_{n}$ with these operators vanishes for $n>0$. Now since operator products of these operators, viewed as cohomology classes, are non-singular, it follows by repeated commutation that all the chiral ground ring elements are primaries of the $N=2$ algebra. However, since they are not Fock vacua, they must appear in the Fock space as secondaries of $N=2$. Hence they are non-vanishing null vectors.

Thus we have seen, in brief, how the $N=2$ twisted superconformal algebra organizes the states of $c=1$ string theory. Tachyons are primaries, discrete states of the same ghost number are secondaries and discrete states of ghost number zero (ground ring generators) are non-vanishing null vectors. A more detailed analysis of this system can be done, to provide the complete classification of $c=1$ string theory physical states in $N=2$ language, and explore its consequences [25]. 


\section{A Special $N=2$ Model}

\subsection{Description of the Coset Model}

In this section we study a special $N=2$ supersymmetric conformal field theory which we argue, here and in following sections, to be equivalent to $c=1$ matter coupled to gravity at non-zero cosmological constant. From the discussion in Section 2, it is clear that such a superconformal model should have untwisted central charge $\hat{c}=3$. The model is a supersymmetric Kazama-Suzuki (KS) coset model [26]

$$
\frac{S \widehat{L(2, R)}}{U(1)}
$$

at level $k=3$ (One can also think of this as $S U(2)$ at level $k=-3$. Here and in the rest of the paper, we use the conventions that for $S U(2), c=3 k /(k+2)$, while for $S L(2, R)$, $c=3 k /(k-2)$.) In this way of writing it we have hidden the fermionic degrees of freedom. It is well known that we can write these in terms of free fermions, which in this case we denote by $b, c$, and the coset can then be thought of as

$$
\frac{S L(2, R) \times U(1)}{U(1)}
$$

The computation of the central charge is

$$
\hat{c}=\frac{1}{3}\left[\frac{3 k}{k-2}+1-1\right]=\frac{3}{3-2}=3
$$

the desired value. The value $k=3$ is the only level for $S L(2, R) \mathrm{KS}$ models which gives $\hat{c}=3$. The supersymmetry currents of the $N=2$ algebra are given by

$$
G^{+}=c J^{+} \quad G^{-}=b J^{-}
$$

where $J^{ \pm}$are the raising and lowering operators of $S L(2, R)$. The $N=2 U(1)$ current $J^{Q}$ is given by

$$
J^{Q}=\frac{k}{k-2} c b-\frac{2}{k-2} J^{3}=3 c b-2 J^{3}
$$

where $J^{3}$ is the third generator of $S L(2, R)$. It is easy to see that with this definition, $G^{ \pm}$ have charges \pm 1 under $J^{Q}$, as they should. The $U(1)$ current $J^{q}$ that we are modding out (i.e., the $U(1)$ in the denominator of (3.1)) is given by

$$
J^{q}=-c b+J^{3}
$$


Note that $J^{q}(z) J^{Q}(0) \sim 0$ as expected (we use $J^{3}(z) J^{3}(0) \sim-k / 2 z^{2}$ ). In the untwisted version of this theory, $b$ and $c$ have spin $1 / 2$. But as discussed in the previous section, only twisted $N=2$ theories are related to the bosonic string. The twisting changes the spin content of the fields by

$$
s \rightarrow s-\frac{Q}{2}
$$

where $Q$ is the $N=2 \mathrm{U}(1)$ charge of the field. This in particular means that the spins of the fields change according to

$$
\begin{gathered}
c: \quad \frac{1}{2} \rightarrow \frac{1}{2}-\frac{k}{2(k-2)}=\frac{1}{2}-\frac{3}{2}=-1 \\
b: \quad \frac{1}{2} \rightarrow \frac{1}{2}+\frac{k}{2(k-2)}=\frac{1}{2}+\frac{3}{2}=2 \\
J^{+}: \quad 1 \rightarrow 1+\frac{1}{k-2}=2 \\
J^{-}: \quad 1 \rightarrow 1-\frac{1}{k-2}=0 \\
G^{+}: \quad \frac{3}{2}-\frac{1}{2}=1 \\
G^{-}: \quad \frac{3}{2}+\frac{1}{2}=2
\end{gathered}
$$

From the spin content of this theory at the particular value $k=3$ we see an amazing similarity to bosonic strings. Namely, the $b, c$ have acquired precisely the right spins to be identified with the usual ghosts of bosonic strings, $G^{+}$is to be identified with the string BRST operator, and $G^{-}$with the usual $b$ ghost. There is a slight puzzle in this correspondence, in that $G^{-}=b J^{-}$contains an extra $J^{-}$piece besides the $b$ ghost. We will return to this point when we discuss the KPZ version of $c=1$ theory in the next section. From Eq.(3.5), $J^{-}$has spin zero. Thus if, as in the KPZ approach, we set it to a constant, we get the precise correspondence with the usual expression in the DDK version of $c=1$ string theory, whose $N=2$ structure was discussed in the previous section.

In order to better understand the connection with the $c=1$ matter theory coupled to gravity, it is important to find the $Q^{+}=\int G^{+}$cohomology of the KS model. Those are the states which are to be identified with physical states of the bosonic theory, since $Q^{+}$is the analog of the BRST operator. In order to do this, it is convenient to go to the Ramond sector of the theory, and then use spectral flow to write the chiral fields which form the cohomology of $Q^{+}$in terms of fields. This is just the familiar story that the Hilbert space 
of the topological theory is best viewed in the Ramond sector but the operators in the NS sector [27]. Note that under spectral flow the $J^{3}$ charge and $c b$ ghost charge $G$ shift respectively by

$$
J^{3} \rightarrow J^{3}+\frac{3}{2} \quad G \rightarrow G+\frac{3}{2}
$$

To construct the states of the $G / H$ coset we start with primary states of the $G$ model, act on them with the $G$ current algebra, and decompose the result into $H$ representations. For us $G$ consists of the $b, c$ system whose representation is just free fermion fock space, and the $S L(2, R)$ Hilbert space. The latter has representations of four types, built on highest weight states (HWS), lowest weight states (LWS), states which are both, or states which are neither. A HWS (LWS) is one which is annihilated by $J_{0}^{+}\left(J_{0}^{-}\right)$:

$$
J_{0}^{+}|H W S\rangle=0=J_{0}^{-}|L W S\rangle
$$

A HWS with $J^{3}=j$ will be denoted $D_{j}^{-}$. Using the $J_{0}^{-}$operator we get the $J^{3}$ eigenvalues $j, j-1, j-2, \ldots$, which form an infinite dimensional representation, unless we encounter a zero vector (which would then correspond to an LWS). A LWS with $J^{3}=j$ will be denoted $D_{j}^{+}$. Using the $J_{0}^{+}$operator we get the $J^{3}$ eigenvalues given by $j, j+1, j+2, \ldots$. We will be primarily interested in the case where $2 j \in \mathbf{Z}$, but will comment on the generalizations below. Representations which have neither HWS nor LWS turn out to have a trivial $Q^{+}$ cohomology, so we will ignore these in the subsequent discussion. The ones which have both can be viewed as HWS (LWS) representations for which the $J_{0}^{-}\left(J_{0}^{+}\right)$operator raised to some power annihilates the state. Thus the raising or lowering terminates at a finite number of steps and gives rise to a finite dimensional representation of $S L(2, R)$.

To study the $Q^{+}$cohomology it is useful to note that

$$
\left\{Q^{+}, Q^{-}\right\}=L_{0}
$$

where $Q^{-}=G_{0}^{-}$and $L_{0}$ is the scaling operator in the Ramond sector (shifted by $-\hat{c} / 8$ ). From this it follows that all the cohomology states of $Q^{+}$are represented by zero eigenstates of $L_{0}$. To see this, suppose to the contrary that there is a cohomology state of $Q^{+}$given by $|\alpha\rangle$ whose $L_{0}$ eigenvalue is $h \neq 0$. Since $|\alpha\rangle$ is a cohomology state, it must be annihilated by $Q^{+}$. Then using (3.7) we deduce that

$$
|\alpha\rangle=\frac{1}{h} Q^{+}\left(Q^{-}|\alpha\rangle\right)=Q^{+}|\beta\rangle
$$


where $|\beta\rangle=\frac{1}{h} Q^{-}|\alpha\rangle$. It follows that $|\alpha\rangle$ is cohomologically trivial. So all the non-trivial cohomology states of $Q^{+}$have zero eigenvalues of $L_{0}$. In a unitary theory an eigenstate of $L_{0}$ with eigenvalue 0 is guaranteed to be cohomologically non-trivial [28] but for a nonunitary theory, like the one we are dealing with, this is no longer true. So we only have a necessary condition for a non-trivial cohomology element. Note that we can have two distinct types of cohomology elements depending on how the $Q^{-}$cohomology is realized. There are $Q^{+}$cohomology states that are annihilated by both $Q^{+}$and $Q^{-}$, and there are others which form pairs of the form $|\psi\rangle$ and $Q^{-}|\psi\rangle$.

Let us see what the condition for vanishing of $L_{0}$ implies. Let us consider a HWS $D_{j}^{-}$. Then the formula for $L_{0}$ of the coset obtained from the primary states of the $G$ theory is given by 26]

$$
L_{0}=\frac{-j(j+1)}{k-2}+\frac{1}{8}+\frac{q^{2}}{k-2}-\frac{\hat{c}}{8}=-j(j+1)+q^{2}-\frac{1}{4}
$$

where $q=j \pm \frac{1}{2}$ is the $U(1)$ charge of $j^{q}$ and the sign refers to which of the spins of the $b, c$ system we take. Note that if we take $q=j+\frac{1}{2}$, i.e. ghost number $-\frac{1}{2}$ then $L_{0}=0$. In fact it is easy to see that this is a non-trivial cohomology state, using the fact that $Q^{+}=c_{0} J_{0}^{+}+\ldots$ It is also annihilated by $Q^{-}$. This gives rise, using spectral flow, to a cohomology state with ghost number

$$
G=-\frac{1}{2}+\frac{3}{2}=1
$$

according to equation (3.6).

Now we search for other states with $L_{0}=0$. We will focus first on states in the coset with $L_{0} \leq 0$, because the states with $L_{0}=0$ can be built on top of such 'ground states'. In a HWS representation, the envelope of states in the $G$ theory can be obtained by either applying $\left(J_{-1}^{+}\right)^{r}$ or $\left(J_{0}^{-}\right)^{s}$ on the highest weight state (see Fig. 1). Let us consider first the $L_{0}$ of states obtained by acting $\left(J_{-1}^{+}\right)^{r}$ on the HWS. It will increase the energy of the $G$ theory by $r$ units and it will shift $q \rightarrow q+r$. Therefore the change in $L_{0}$, starting from $L_{0}=0$ is

$$
\Delta L_{0}=L_{0}=r+(q+r)^{2}-q^{2}=r(2 q+1)+r^{2}=r((2 j+2)+r)
$$

Since $r \geq 0$ the condition that this gives $L_{0} \leq 0$ is that

$$
j \leq-1 \quad \text { and } \quad 0 \leq r \leq-2 j-2
$$


For these values of $j$ and $r$, the ground state of the coset has negative dimension. By using the other raising operators we can obtain states with $L_{0}=0$ only for this range of $j$ and $r$. Considering now the $\left(J_{0}^{-}\right)^{s}$ action, we get

$$
\Delta L_{0}=L_{0}=(q-s)^{2}-q^{2}=-2 q s+s^{2}=s(s-2 j-1)
$$

and the condition for this $L_{0}$ to be negative is that

$$
j \geq \frac{-1}{2} \quad \text { and } \quad 0 \leq s \leq 2 j+1
$$

We thus see that for a fixed $j$, we have only a finite number of potential candidates for the cohomology states of $Q^{+}$, because there are only finitely many combinations of raising operators that can raise the energy to $L_{0}=0$.

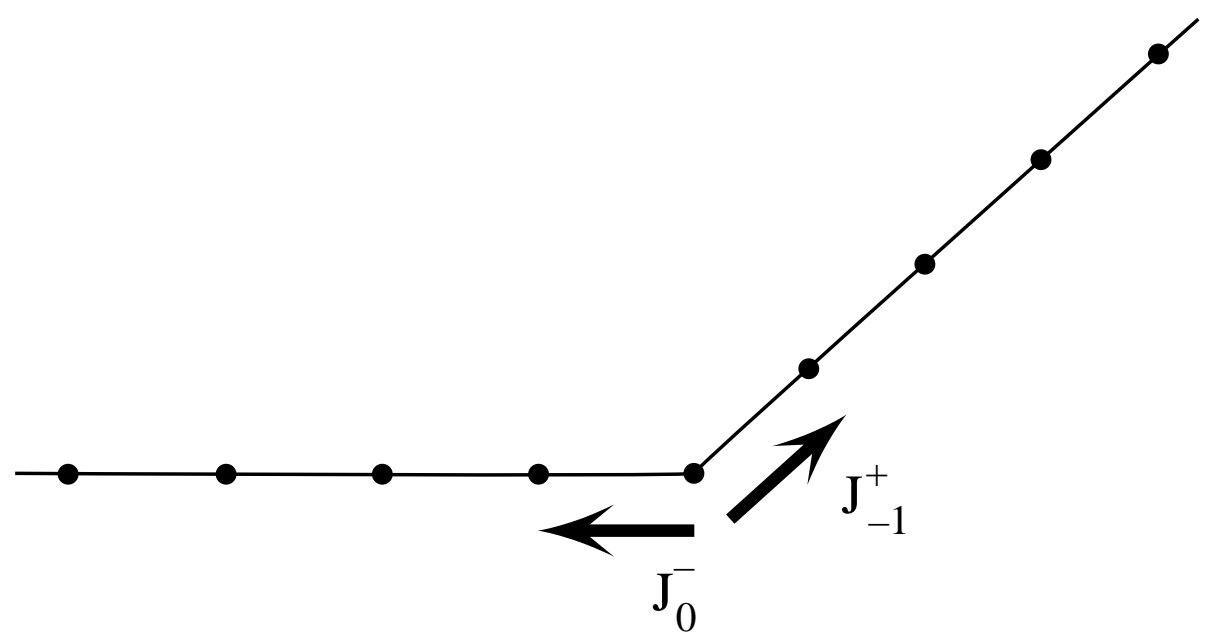

Fig. 1: The envelope of states in a HWS representation is obtained by acting with $J_{-1}^{+}$or $J_{0}^{-}$on the highest state.

We have carried out this discussion for a HWS $D_{j}^{-}$. By a CPT transformation a similar story holds for a LWS $D_{-j}^{+}$, where we have to flip the signs of all charges in the process. In particular the LWS itself will correspond to a non-trivial cohomology element with ghost number $G=2$. Similarly using $\left(J_{-1}^{-}\right)^{r}$ and $\left(J_{0}^{+}\right)^{s}$ we get a finite range of values of $j, r$ and $j, s$ with negative $L_{0}$ just as in (3.10) and (3.12).

To proceed further we have to discuss which representation of affine $S L(2, R)$ KacMoody algebra we should use. There are 4 natural choices: the irreducible representation $I$, which is natural for $k<0$ (which can be thought of as $S U(2), k>0$ ), the Wakimoto representation $W$, the dual Wakimoto representation $W^{*}$ and the Verma module $V$. The 
one which turns out to be most clearly related to $c=1$ theory coupled to gravity is $W$, though there are some intriguing features for the other representations which we mention later.

To begin with we recall how the Wakimoto fields realize the $S L(2, R)$ algebra. There are three bosonic fields, $\beta, \gamma$ and $\phi$. The $S L(2, R)$ currents are given by

$$
\begin{gathered}
J^{+}=\beta \gamma^{2}-\sqrt{2} \gamma \partial \phi+3 \partial \gamma \\
J^{3}=\beta \gamma-\frac{1}{\sqrt{2}} \partial \phi \\
J^{-}=\beta
\end{gathered}
$$

In the untwisted theory $\beta$ has dimension 1 and $\gamma$ has dimension zero, but in the twisted theory which is the one we are interested in, it is easy to see that $\beta$ will have spin 0 , and $\gamma$ spin 1. They satisfy the $\operatorname{OPE} \gamma(z) \beta(0) \sim-1 / z . \phi$ is a scalar field with a background charge. In the twisted theory the background charge of $\phi$ is exactly the same as that of the Liouville field in $c=1$ theory, and we will later identify it with that.

So far we have a good description of the field content of the $S L(2, R)$ theory and the fermionic $b, c$ system. Now we need an equally convenient representation for the coset model. This is readily obtained by gauging the $J^{q}$ current. As is familiar [29], this leads to the introduction of a scalar field $X$, whose derivatives are related to the gauge field, and a fermionic ghost system $B, C$ of dimension 1,0 . These ghosts can be used to define the BRST operator for the $U(1)$ gauge symmetry:

$$
Q_{U(1)}=C\left(J^{3}-c b-i \frac{\partial X}{\sqrt{2}}\right)=C\left(\beta \gamma-c b-\partial X^{-}\right)
$$

where

$$
X^{ \pm}=\frac{\mp i X+\phi}{\sqrt{2}}
$$

Note that the $X$-field is a $c=1$ system whose radius is fixed by the level of the $j^{q}$, which is one, to be the self-dual value. Let us summarize what we have found as the Hilbert space of the coset:

$$
\mathcal{H}=[\phi]+[X]+[b, c]+[\beta, \gamma]+[C, B]
$$

Note that this differs from the standard DDK Hilbert space of $c=1$ only by the appearance of bosonic $(\beta, \gamma)$ and fermionic $(C, B)$ fields of spins $(0,1)$. In order to make contact with 
KS theory we have to reduce the Hilbert space of this theory by first imposing the $U(1)$ constraint via the BRST operator $Q_{U(1)}$ :

$$
\mathcal{H}_{K S}=\mathcal{H}_{Q_{U(1)}}
$$

Then we can consider the cohomologies of the KS theory on this reduced Hilbert space by acting with

$$
G^{+}=c J^{+}=c\left(\beta \gamma^{2}-\sqrt{2} \gamma \partial \phi+3 \partial \gamma\right)
$$

In this representation $G^{-}$is particularly simple:

$$
G^{-}=b \beta
$$

Note that using the constraint (3.14), the $N=2$ current $J^{Q}(3.3)$ can be written as

$$
J^{Q}=c b-i \sqrt{2} \partial X
$$

This is precisely what is expected for $c=1$ theory based on our analysis in section 2 (see (2.1) with $\eta=X$ and $y=i \sqrt{2}$ ).

The cohomology spectrum of this KS theory is analyzed by E. Frenkel in appendix A, using techniques developed in [30]. Let us consider a HWS $D_{j}^{-}$of Wakimoto. His results are as follows: Apart from the cohomology we discussed before, there is precisely one pair of cohomology states of ghost number $(0,1)$ for the range of $r$ and $j$ given by (3.10) (with $q=j+r+1 / 2)$ and one pair of cohomology states of ghost number $(1,2)$ for the range of $r$ and $j$ given by (3.12) (with $q=j-s+1 / 2$ ) (see Fig. 2). Moreover, as we will see explicitly below, the higher ghost number cohomology state gets mapped by $Q^{-}$to the lower one. This is not surprising in view of the fact that $Q^{-}$cohomology, being quadratic in fields, is non-trivial only for the HWS itself. For the LWS $D_{-j}^{+}$apart from the ghost number 2 cohomology state that we mentioned before there is a pair of cohomology elements with $r$ and $j$ satisfying condition (3.10) (with $q=-j-r-1 / 2$ ) with ghost numbers $(2,3)$ and the ones satisfying condition (3.12) (with $q=-j+s-1 / 2)$ with ghost numbers $(1,2)$. In both classes the highest ghost number state is mapped to the lower one by the action of $Q^{-}$, as discussed before.

In the next two subsections, we will explicitly construct representatives of the cohomology of this coset model, and display their similarity to those of the $c=1$ string. 

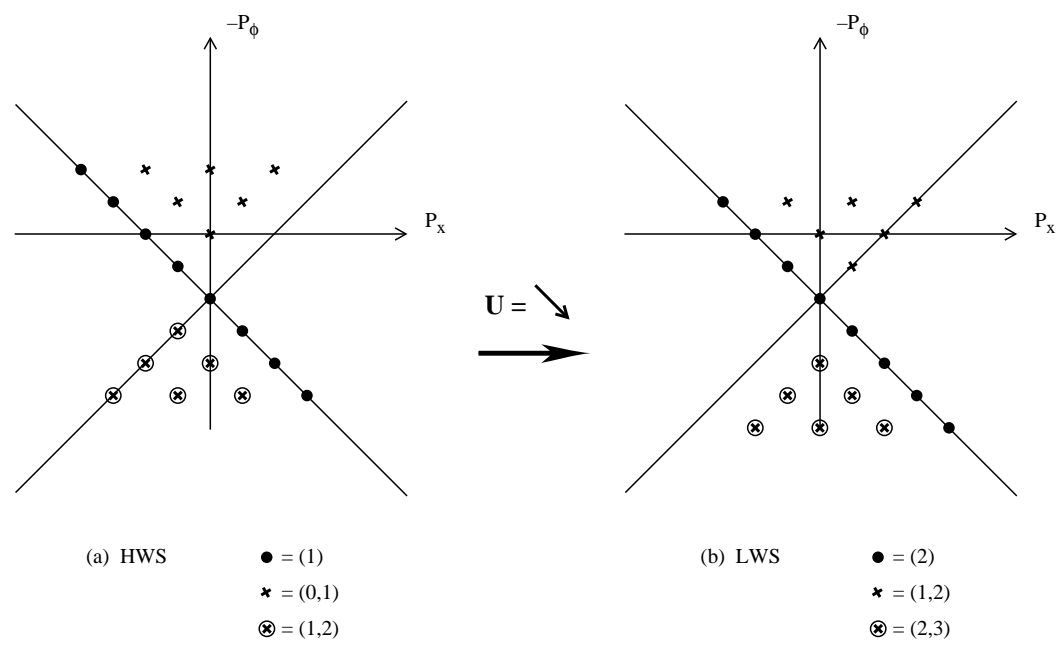

Fig. 2: The cohomology spectrum for HWS (a) and LWS (b). The spectrum of LWS can be obtained from that of HWS by operating with $U$ which shifts the matter and Liouville momenta as shown, and increases ghost number by one. The degeneracy and ghost numbers of the cohomology states are represented in the figure. A given representation leads to states in the same horizontal line. The cosmological constant operator in (a) corresponds to $D_{-1 / 2}^{-}$with $j$ increasing as we go down in the figure. The cosmological constant state in (b) corresponds to $D_{1 / 2}^{+}$ with $j$ increasing as we go up in the figure. Note that the positive Liouville momentum in our convention corresponds to the lower part of the figures.

\subsection{Explicit Construction of the Cohomology: Highest Weight States}

Let us first start with the Hilbert space generated by HWS. The simplest cohomology states, as we discussed before, arise from the highest weights themselves. The cohomology state considered below Eq. (3.8) (coming from $D_{j}^{-}$with $q=j+1 / 2$ ) corresponds to the field

$$
V_{j}=c \exp \sqrt{2}\left[\left(j+\frac{1}{2}\right) i X+\left(j+\frac{3}{2}\right) \phi\right]=c \exp \left[(2 j+1) X^{-}+\sqrt{2} \phi\right]
$$

To see this, note that the exponent of $\phi$ is $j+3 / 2$ because the spectral flow shifts the $J^{3}$ eigenvalue by $3 / 2$. Also, we started with a ghost number $-1 / 2$ state and spectral flow shifts that by $3 / 2$ to ghost number 1 , thus explaining the appearance of $c$ in the above. The highest weight vacuum state is defined as the state annihilated by $\gamma_{0}$ and all higher modes, and $\beta_{1}$ and all higher modes. Since in the twisted theory $\gamma$ and $\beta$ have spins 1,0 respectively, this is the vacuum given by the path integral, and requires no further insertions. The above expression for these cohomology states are precisely the same expressions as appear in $c=1$ DDK theory for tachyon states of ghost number 1 . 
The above tachyon states for all $j$ have a particular chirality (from the target space point of view), as they depend on $X^{-}$and not on $X^{+}$.

To make the contact with DDK theory more explicit, let us re-label these states as follows. For $j \leq-1 / 2$, define $s=-(j+1 / 2)$ and denote the corresponding states

$$
\tilde{Y}_{s,-s}^{+}=c e^{-\sqrt{2} i s X} e^{\sqrt{2}(1-s) \phi}, \quad s \geq 0
$$

For $j \geq-1 / 2$, define $s=j+1 / 2$ and denote the state

$$
\tilde{Y}_{s, s}^{-}=c e^{\sqrt{2} i s X} e^{\sqrt{2}(1+s) \phi}, \quad s \geq 0
$$

These states can now be compared with those of Ref. [10] corresponding to the same labels with the tildes dropped.

How do we find the rest of the states? One series of states is easy to find. From the cohomology computation in the appendix, it is clear that one state of ghost number 1 arises with the $X$ momentum exactly that allowed by the boundary of the conditions (3.10) and (3.12). Since there is exactly one state in our theory with that $X$ momentum and with ghost number 1 and dimension zero, it is straightforward to identify it. For HWS with $j \leq-1$, we set $r=-2 j-2$, which is the boundary of Eq.(3.10). The state is obtained by acting with $\left(J_{-1}^{+}\right)^{2|j|-2}$ on the HWS. Using (3.13), the action of this operator on the HWS is equivalent to the action of $\gamma_{-1}^{2|j|-2}$. So the state coming from the boundary of (3.10) with $j \leq-1$ is

$$
B_{j}=c \gamma^{2|j|-2} \exp \left(\sqrt{2}\left(j+\frac{3}{2}\right) \phi\right)+\left(i \sqrt{2}\left(-j-\frac{3}{2}\right) X\right)=c \gamma^{2|j|-2} \exp \left((2 j+3) X^{+}\right)
$$

where we have used the $U(1)$ constraint (3.3) to find the $X$ momentum, and also the fact that in the twisted theory $\gamma$ has dimension 1. It is easy to check directly that these fields commute with $Q^{+}$and are not $Q^{+}$trivial. They correspond to ghost number 1 discrete states of $c=1$ closest to the positive $X$ momentum and negative Liouville momentum (see fig. 2).

Again it is convenient to re-label these states to display the correspondence with DDK theory. The case $j=-1$ has already been counted in Eq.(3.18), so take $j \leq-3 / 2$ and let $s=-(j+3 / 2)$. Then, define

$$
\tilde{a} \tilde{\mathcal{O}}_{s, s}=c \gamma^{2 s+1} e^{-2 s X^{+}}
$$


The notation and the precise correspondence of these states with Ref. 110 will become clear shortly.

As discussed before, the $Q^{-}$cohomology is trivial on these states, so we can obtain another set of $Q^{+}$cohomology states just by acting on these with $Q^{-}=G_{0}^{-}$. This is given by the residue of the double pole of the OPE of $b \beta$ with the above, which is $($ for $j<-1$ )

$$
B_{j}^{\prime}=\gamma^{2|j|-3} \exp \left((2 j+3) X^{+}\right)
$$

With the same definition of $s$ above, these states may be re-labelled

$$
\tilde{\mathcal{O}}_{s, s}=\gamma^{2 s} e^{-2 s X^{+}}
$$

They have ghost number zero and should generate a subset of the ground ring. In particular we see that the identity operator is already in the list, corresponding to $j=-3 / 2$. The state which has the right momentum to correspond to the ground ring element $x$ of $c=1$ theory [10] comes from $j=-2$ :

$$
\tilde{x}=\tilde{\mathcal{O}}_{\frac{1}{2}, \frac{1}{2}}=\gamma \exp \left(-X^{+}\right)
$$

Moreover all the rest of the above ghost number zero states can be written as powers of this element:

$$
B_{j}^{\prime}=\gamma^{2|j|-3} \exp \left((2 j+3) X^{+}\right)=\tilde{x}^{2|j|-3}
$$

corresponding to

$$
\tilde{\mathcal{O}}_{s, s}=\tilde{x}^{2 s}
$$

Thus the ring structure is exactly the same as that generated by $x$ in $c=1$ DDK theory.

A natural question is whether $\tilde{x}$ and $x$ are related. Indeed they are: on modifying the $\tilde{x}$ generator by adding $Q_{U(1)}$ trivial fields, we get

$$
\begin{gathered}
\tilde{x} \sim \gamma \exp \left(-X^{+}\right)-\left\{Q_{U(1)}, B \beta^{-1} \exp \left(-X^{+}\right)\right\}=\beta^{-1}\left(c b+\partial X^{-}\right) \exp \left(-X^{+}\right)= \\
=\beta^{-1} x
\end{gathered}
$$

where we have been somewhat formal in taking negative powers of $\beta$. Since $\beta$ has dimension 0 this is not such an unnatural thing to do. We will make this more precise below when we bosonize the $\beta, \gamma$ system. At any rate, on the Hilbert space omitting the kernel of $\beta$ we see that the two are related. 
Now we come to the question of how to find the other discrete states coming from HWS. In the $c=1$ theory they can be obtained by acting with the $S U(2)$ raising and lowering operators which are present at the self-dual radius. There is a similar symmetry in our case, which follows from an application of the ideas in [10]. The idea in the context of bosonic strings is to generate a $(1,0)$ current from the $(0,0)$ cohomology states by acting with $b_{-1}$. The analog of this operator for us is $G_{-1}^{-}$, which means we consider the OPE of $b \beta$ with the fields and take the residue of the single pole term. Applying this to the tachyon state $V_{-3 / 2}$ (which in $c=1$ gives rise to the lowering current $\exp (-i \sqrt{2} X)$ ) we obtain

$$
K^{-}=\oint b \beta \cdot(c \quad \exp (-i \sqrt{2} X))=\beta \exp (-i \sqrt{2} X)
$$

The minus superscript over $K^{-}$is to remind us that the corresponding charge decreases the $X$ momentum by one unit. The operator $K^{-}$is the analog of the lowering operator of the $c=1$ theory, but it contains an extra factor of $\beta$. Using $K^{-}$we can generate all the discrete states with ghost number 0 (and the corresponding ghost number 1 states which can be mapped to them by $Q^{-}$). We apply $K^{-}$up to $2 s$ times on the states corresponding to $\tilde{\mathcal{O}}_{s, s}$ The states so obtained are denoted

$$
\tilde{\mathcal{O}}_{s, n}=\left(K^{-}\right)^{s-n} \tilde{\mathcal{O}}_{s, s}
$$

As an example, let us consider $K^{-}$acting on $\tilde{x}$ to obtain the analog of the $y$ generator. We find that it is given by

$$
\tilde{y}=\int \beta \exp (-i \sqrt{2} X)\left(\gamma \exp \left(-X^{+}\right)=(\beta \gamma-i \sqrt{2} \partial X) \exp \left(-X^{-}\right)\right.
$$

Using the $U(1)$ constraint, this is easily seen to be equivalent to

$$
\tilde{y}=\left(c b+\partial X^{+}\right) \exp \left(-X^{-}\right)=y
$$

which is exactly the same expression as in the DDK theory. We could have obtained this answer equivalently by applying $K^{-}$on $\beta^{-1} x$, which would reduce the computation to exactly the one which gives rise to $y$ in the usual $c=1$ theory (as $\beta$ in $K^{-}$plays no role in the OPE, and just cancels the factor of $\beta^{-1}$ ). The subspace of the chiral ring generated by $[\tilde{x}, \tilde{y}]$ is thus isomorphic to the ring generated by $\left[\beta^{-1} x, y\right]$, which is in turn the same as the $c=1$ ring $C[x, y]$ because $x$ and $y$ have no $\beta$ excitations in them. We thus obtain the exactly the same expression as in DDK theory for all discrete states of ghost number 
0 , modulo factors of $\beta^{-1}$ which accompany each factor of $x$. As we discussed, the ghost number 1 analogs of these states can be obtained by applying $K^{-}$to $D_{j}$ a number of times.

There is another way of doing this, which makes the connection with $c=1$ DDK theory more transparent. Note that the ghost number one state paired with the identity is

$$
D_{-3 / 2}=c \gamma
$$

In the DDK theory this is given by an operator $a$ [10 defined by

$$
a=\left\{Q, \frac{\phi}{\sqrt{2}}\right\}
$$

It turns out that if we use our expression for $Q^{+}$, we find

$$
\tilde{a}=\left\{Q^{+}, \frac{\phi}{\sqrt{2}}\right\}=c \gamma
$$

Note that $\tilde{a}^{2}=0$ and $a$ commutes with $Q^{+}$but not with $Q^{-}$:

$$
\left\{Q^{-}, c \gamma\right\}=1
$$

This implies that we can obtain the pairs of discrete states simply by multiplying the one of lower ghost number with $\tilde{a}=c \gamma$, which increases the ghost number by 1 . This explains the notation in Eq.(3.21).

So far we have discussed the discrete states coming from HWS with $j \leq-1 / 2$. For HWS with $j \geq 0$ we can again use the tachyon states $V_{j}$ and act on them with $K^{-}$a number of times to find all the discrete states with ghost number 1. Since they are obtained from the tachyon state $V_{j}$ which is annihilated by $Q^{-}$, and $K^{-}$commutes with $Q^{-}$, it follows that $Q^{-}$also annihilates all these states. So the pair which they form must be with a ghost number 2 discrete state, obtained by acting on them with $\tilde{a}$, as discussed before. Thus we have

$$
\tilde{Y}_{s, n}^{-}=\left(K^{-}\right)^{s-n} c e^{\sqrt{2} i s X} e^{\sqrt{2}(1+s) \phi}, \quad-s \leq n \leq s
$$

and their partners $\tilde{a} \tilde{Y}_{s, n}^{-}$, for which, however, the range is $-s \leq n \leq s-1$. The reason is that $\tilde{a}$ has a vanishing OPE with $\tilde{Y}_{s, s}^{-}$.

Note that the state with the maximum number of $K^{-}$operators acting on $V_{j}$ is the one which saturates (3.12). This, however, corresponds to a tachyon state with the opposite 
chirality. Noting that $J_{0}^{-}$is represented by $\beta$, and taking into account (3.14), we find that this tachyon is given by

$$
\begin{gathered}
\tilde{V}_{j}=\beta^{2 j+1} c \exp \left[\sqrt{2}\left(-i\left(j+\frac{1}{2}\right) X+\left(j+\frac{3}{2}\right) \phi\right)\right] \\
=\beta^{2 j+1} c \exp \left[(2 j+1) X^{+}+\sqrt{2} \phi\right]
\end{gathered}
$$

where $j \geq 0$. In the notation of Eq.(3.27), this is just $\tilde{Y}_{s,-s}^{-}$, which has the same expression as in $c=1$ DDK theory except for some extra $\beta$ dependence. Note that we can similarly obtain the tachyon state with positive chirality of ghost number 2 by acting with $\tilde{a}$ which gives $\beta^{2 j} c \partial c \exp \left[(2 j+1) X^{+}+\sqrt{2} \phi\right]$.

We have thus finished discussing how we can explicitly obtain all the cohomologies coming from HWS depicted in fig. 2 in the Wakimoto representation. Note that we have obtained all the tachyon states (of both ghost numbers) with positive Liouville dressing and positive chirality, and half of the tachyon states with negative chirality (those with ghost number 1). Of the discrete states, we have found pairs with ghost numbers $(0,1)$ and negative Liouville dressing, and pairs with ghost numbers $(1,2)$ and positive Liouville dressing (see fig. 2). These results may be summarized as follows:

$$
\begin{aligned}
& \text { (i) } \quad Y_{s,-s}^{+}=c \exp (-i \sqrt{2} s X+\sqrt{2}(1-s) \phi) \quad(s \geq 0 .) \\
& \text { (ii) } \quad Y_{s, n}^{-}=\left(K^{-}\right)^{s-n} \cdot c \exp (i \sqrt{2} s X+\sqrt{2}(1+s) \phi) \quad(-s \leq n \leq s) \\
& \text { (iii) } \quad \tilde{a} Y_{s, n}^{-}, \quad(-s \leq n \leq s-1) \\
& (\text { iv }) \quad O_{s, n}=\beta^{-(s+n)} x^{(s+n)} y^{s-n} \quad(s \geq 0 \quad,-s \leq n \leq s) \\
& (v) \quad \tilde{a} O_{s, n} \quad(s \geq 0 \quad,-s \leq n \leq s)
\end{aligned}
$$

where $x, y$ are as in the $c=1$ DDK theory, $s$ and $n$ are both integer or half-integer, $K^{-}=\int \beta e^{-\sqrt{2} i X}$, and $\tilde{a}=c \gamma$.

The rest of the states come from LWS representations, to which we now turn.

\subsection{Explicit Construction of the Cohomology: Lowest Weight States}

In obtaining the cohomology states from LWS we can use the same ideas as above. However, it turns out that there is an even faster way of getting all these states: The HWS and LWS representations turn out to be equivalent in the coset language. This follows from the fact that if we have a coset of the form $G / H$ the common center of $G$ and $H$ generate an isomorphism between coset characters [31 28]. In the case at hand the action 
on $S L(2, R)$ is to take a HWS of spin $j$ and map it to LWS of spin $j+(k / 2)$. For us, this means that

$$
D_{j}^{-} \rightarrow D_{j+\frac{3}{2}}^{+}
$$

In addition, the ghost number and the $U(1)$ charge are increased by one unit. In the Wakimoto realization, this is the spectral flow by the operator

$$
U=c \delta(\beta) \exp \left(X^{-}\right)
$$

where $\delta(\beta)$ (which also contributes +1 to the $U(1)$ constraint) is needed to change the HWS to a LWS (annihilated by $J^{-}$). The operator $U$ can be viewed as the spectral flow generated by the constraint current (3.14). From that viewpoint, it may be natural to assume that we have to identify the two representations and that they should be viewed as picture-changed versions of one another. Taking into account the shift in the ghost number and the $X$ and $\phi$ momenta obtained by acting on HWS with $U$, we immediately get the spectrum of cohomologies depicted in fig. 2. Note in particular that if we put the two spectra together we end up with precisely the same degeneracy of cohomology classes as in the $c=1 D D K$ theory if we do not identify the two representations! We will have more discussion on this below.

Just to give a sample of the LWS that we obtain, we write the tachyon states coming from the LWS representation $D_{j}^{+}$which are of ghost number 2 :

$$
T_{j}=c \partial c \exp \left[(2 j-1) X^{-}+\sqrt{2} \phi\right] \quad \delta(\beta)
$$

We can also obtain the ghost number 3 partners of these states by multiplying by $\tilde{a}=c \gamma$ as was the case for HWS.

We also write explicit expressions for tachyon states with negative Liouville dressing and opposite chirality, which are obtained from LWS with $j \leq 0$ (these can be obtained by multiplying $\tilde{x}^{n}$ by $U$ or by acting on $T_{j}$ (with $j \leq 0$ ) with $\left(J_{0}^{+}\right)^{2|j|+1}$, which on LWS is equivalent to acting with $\left.\gamma_{0}^{2|j|+1}\right)$ :

$$
\tilde{T}_{j}=c \partial c \exp \left[(2 j-1) X^{+}+\sqrt{2} \phi\right] \delta^{2|j|+1}(\beta)
$$

where the superscript over $\delta$ denotes the order of derivative of the delta function. We can use $Q^{-}$, which does not annihilate these states, to obtain the corresponding ghost number 1 tachyon states. We can now obtain all the ghost number 1 and 2 discrete states by considering $U \tilde{x}^{n} \tilde{y}^{m}$ and $c \gamma U \tilde{x}^{n} \tilde{y}^{m}$. 
As an example, let us consider the case $j=-1 / 2$, and write the corresponding discrete states. In this case there is only one pair of discrete states, and they are found by the above method to be

$$
R=i \sqrt{2} c \delta(\beta) \partial X+c \partial \beta \delta^{\prime}(\beta) \quad \text { and } \quad \tilde{a} R
$$

Note that the state $R$ is the analog of the discrete state in the ordinary $c=1$ DDK theory at zero cosmological constant given by $c \partial X$.

If we view the coset model as arising from a gauged WZW model, then the natural question is whether we can realize the Wakimoto fields in terms of the field variables of the WZW theory. It was shown in [32] that this is indeed possible. There is one interesting subtlety: The Wakimoto fields have one extra degree of freedom relative to the fields of the WZW theory, and this leads to the appearance of a screening charge given by

$$
Q_{F}=\oint \beta \exp (\sqrt{2} \phi)
$$

(and similarly for the right-movers). The existence of this screening current was seen to be crucial in computations in WZW theory [32] in the Wakimoto realization (and in particular needed to balance the $\phi$ charge violation). The cosmological constant operator in the ghost number 1 representation is given by $V_{-1 / 2}=c \exp \sqrt{2} \phi$. When acted on by $G_{-1}^{-} \bar{G}_{-1}^{-}$, which is needed in order to add it to the action, gives us the current

$$
G_{-1}^{-} \bar{G}_{-1}^{-} V_{-1 / 2}=\beta \bar{\beta} \exp (\sqrt{2} \phi),
$$

Thus, the existence of the above screening charge insertion is directly related to the fact that the cosmological constant is non-zero for us, i.e., the screening current of the theory automatically puts cosmological constant operator insertions in correlation computations in order to achieve $\phi$ momentum conservation. Moreover the identification of the above operator with the cosmological constant operator of $c=1$ DDK theory suggests that if we set $\beta$, which is a dimension zero operator, to a constant:

$$
\beta=\bar{\beta}=\sqrt{\mu}
$$

(where $\mu$ is the cosmological constant) then we should obtain an exact correspondence with $c=1$ DDK theory. We will see in the next section that (3.32) is the precise condition dictated by the KPZ formulation. Again, we see that setting $\beta$ to a constant makes the 
correspondence with $c=1$ theory exact. Put differently, if we count the number of times we use the screening current, and if each time we use the (left and right) currents we put a factor of $\mu$, we get the correct $\mu$ dependence. As it stands the KS theory counts each insertion of screening operator with weight one and should be viewed as having $\mu=1$.

Even though setting $\beta$ to a constant appears to be relevant for comparison to the DDK theory, at first sight there seems to be an obstacle: The LWS all involve $\delta(\beta)$, and therefore setting $\beta=\sqrt{\mu}$ will disallow all these states. Thus we will miss half of our states. Moreover, ignoring the LWS means that we are getting rid of the dual fields of HWS. In particular this would imply that the two point functions which pair a HWS with a LWS do not exist, which does not make much sense.

However, it has been observed [33] that dual fields to HWS in the Wakimoto representation can be constructed without the use of LWS if we allow negative powers of some fields. In our case this can be done if we allow negative powers of $\beta$. In fact at the level of fields, rather than states in the Hilbert space, it makes sense to talk about the cohomology of chiral fields. Allowing negative powers of $\beta$ gives rise to new cohomology fields which are in one-to-one correspondence with those of LWS, and can be obtained from them by the simple substitution

$$
\delta(\beta) \rightarrow \beta^{-1}
$$

It is easy to see that these are in the cohomology and satisfy the (3.3) constraint. The fields thus obtained will not correspond to states in the Hilbert space with well defined $S L(2, R)$ quantum numbers, but nevertheless they are natural for us, as they were in the computations of $S L(2, R)$ correlation functions in [33].

In order to make this a little more precise, it turns out to be useful to bosonize the $\beta, \gamma$ system [34] in terms of two scalar fields $u, v$ by

$$
\beta=\exp (u-i v) \quad \gamma=-i \partial v \exp (-u+i v)
$$

where $u, v$ have background charges (contributing to the energy momentum tensor in the form $\left.\left(-\partial^{2} u+i \partial^{2} v\right) / 2\right)$. In this form, the inverse powers of $\beta$ make sense:

$$
\beta^{-1}=\exp (-u+i v)
$$

Noting that we need an extra $\beta^{-1}$ to absorb the $u, v$ background charge, it is an easy check that for instance $V_{j}$ and $T_{j}$ (with the substitution (3.33)) give a non-zero two point function, as do $\tilde{V}_{j}$ and $\tilde{T}_{j}$. 
With the substitution (3.33) and after setting $\beta$ to a constant (which we discuss in the next section), we see that all the states of the $\mathrm{KS}$ model are identically represented as in the $c=1 D D K$ theory. This completes the correspondence with $c=1$ states

Returning to the construction of the dual states to the HWS, we can simply act on the HWS states in Eq.(3.28) with U given by (3.29), and make the replacement (3.33). This is equivalent to multiplying by $c \beta^{-1} \exp \left(X^{-}\right)$and taking the regular term in the OPE (this is also true for the usual $c=1$ DDK theory except for the extra factor of $\beta^{-1}$ ). Some of the fields so obtained are:

$$
\begin{aligned}
& a Y_{s, s}^{-}=c \partial c \beta^{-1} \exp (\sqrt{2} i s X+\sqrt{2}(1+s) \phi) \quad(s \geq 0) \\
& a Y_{s, n}^{+}=\left(K^{-}\right)^{s-n} \cdot c \partial c \beta^{-2 s-1} \exp (\sqrt{2} i s X+\sqrt{2}(1-s) \phi(-s \leq n \leq s) \\
& Y_{s, n}^{+}=\left(K^{-}\right)^{s-n} \cdot c \beta^{-2 s} \exp (\sqrt{2} i s X+\sqrt{2}(1-s) \phi) \quad(-s \leq n \leq s)
\end{aligned}
$$

and the rest can be constructed similarly.

We thus see explicitly that except for extra factors of $\beta$ (which go away if we set $\beta=1$ ), the states which are in the cohomology of $Q^{+}$(relative to $Q^{-}$) have exactly the same expressions as in the $c=1$ DDK theory.

Discrete states of ghost number 1 (which are annihilated by $b_{0}$ ) were used in 10 to construct an area-preserving symmetry algebra for the ring generated by $x$ and $y$ for the $c=1$ model at zero cosmological constant. For us, the corresponding object would be obtained by acting with $G_{-1}^{-}$on the ghost number 1 discrete states coming from LWS, with the substitution (3.33), and on the tachyon states $V_{j}$ from the HWS. It is easy to see that their action on the ground ring is given by area-preserving diffeomorphisms exactly as for the usual $c=1$ case [10 11]. This is obvious in view of the fact that the only difference here with respect to the $c=1$ case is some extra $\beta$ dependence, which turns out not to have any effect on the algebra.

For example, the $S U(2)$ subalgebra of the area-preserving symmetry is represented here by

$$
W_{1,1}^{+}=-\beta^{-1} \exp (\sqrt{2} i X)
$$

1 The discussion of cohomology states was only chiral (for left-movers). As the story for combining the left and right movers is similar to $\mathrm{c}=1$, we did not bother to discuss this point. In particular the neutrality of the Hilbert space under $G_{0}^{-}-\bar{G}_{0}^{-}$implies that only $\tilde{a}+\overline{\tilde{a}}$ can appear when we combine left and right movers, which is the same thing that happens in $c=1 \mathrm{DDK}$ theory [10]. 


$$
\begin{gathered}
W_{1,0}^{+}=-\beta^{-1} \partial \beta+\sqrt{2} i \partial X \\
W_{1,-1}^{+}=\beta \exp (-\sqrt{2} i X)
\end{gathered}
$$

Note in particular that the current responsible for the change of radius, $W_{1,0}^{+}$, now has an extra $\beta$-dependent piece.

This completes the construction of explicit representatives of the cohomology in the Wakimoto representation.

\subsection{More About the Coset Model}

Let us briefly mention what happens if we consider instead of the Wakimoto representation $W$, other representations of affine $S L(2, R)$. We first consider the dual Wakimoto representation $W^{*}$, which turns out to be rather interesting. Choosing $W^{*}$ means that we choose the same fields as before but interchange $+\leftrightarrow-$ which in particular means that $\beta=J^{+}$(and some signs are changed in (3.13)). This means that now the spin of $\beta$ is 2 and that of $\gamma$ is -1 . Moreover the field $\phi$ in this representation has no background charge!

So the spectrum for this theory will consist of two scalar fields $X$ and $\phi$ with no background charge, two sets of ghost fields $(b, c)$ and $(\beta, \gamma)$ of spins $(2,-1)$, one fermionic and one bosonic, and a pair of fermions of spin $(0,1)$. Amazingly, this is the same field content as topological gravity coupled to $\hat{c}=1$ topological matter! To make this a little more transparent let us define a complex scalar field coordinatizing an infinite cylinder

$$
Z=\frac{(\phi+i X)}{\sqrt{2}}
$$

and redefine the fermionic fields $B, C$ by

$$
\psi^{*}=C \quad \psi=B
$$

In addition we have two BRST charges $Q^{+}$and $Q_{U(1)}$ which now take the form

$$
Q^{+}=c \beta \quad Q_{U(1)}=-\psi^{*}(\partial Z+c b-\beta \gamma)
$$

Solving the corresponding cohomology in this case is much easier than in the case of $W$ because the fields appear only quadratically in the BRST charges. In particular considering the $Q^{+}$cohomology means eliminating $b, c, \beta$ and $\gamma$, and so $Q_{U(1)}$ reduces to

$$
\psi^{*} \partial Z
$$


whose cohomologies are of the form

$$
\exp (n Z)
$$

for integer $n$. (The fact that $n$ should be integer follows from the radius of the $X$ field). Precisely this topological theory was conjectured to be related to the $c=1$ bosonic matter theory in [35]. The spectrum of the theory in this language seems to be smaller than what is required for $c=1$. However, it is conceivable that the rest of the discrete states in this set up correspond to gravitational descendants. It would be extremely interesting to develop this further.

If we work with the irreducible representation $I$ of $S L(2, R)$, obtained by deleting the null states $⿴$, it turns out that we get exactly the same states as before except that the boundary states for HWS with ghost number 1 and for LWS with ghost number 2 will be missing (see appendix A). If we work with Verma module $V$ of the current algebra, then for HWS the cohomology for $j<-1 / 2$ matches that of the $W^{*}$ module and for $j \geq-1 / 2$ it matches that of $W$, which is a possibly interesting mixture of these two cases (see appendix A).

Before concluding this section let us note that it is relatively easy to change the radius from the self-dual value to an integer $n$ times the self-dual one. One simply considers an $n$-fold cover of the $S L(2, R)$ theory, which means that the allowed $j$ 's are multiples of $1 / 2 n$. It is amusing that precisely for these values of radii the existence of a Penner-like model was conjectured in [15] and found in [36] (See also other works on the Penner model [37]).

\section{KPZ Version of $c=1$}

In the last section we saw that the $S L(2, R) / U(1)$ Kazama-Suzuki model at level $k=3$ is very similar to the $c=1$ system coupled to gravity, and it becomes isomorphic to it if we set $\beta=\sqrt{\mu}$. In this section we will see that the correspondence is more transparent if we use the KPZ formulation of non-critical matter coupled to gravity [20]. In this formulation, which can also be used to deal with the case where the cosmological constant is not zero, the Liouville system is represented by a twisted $S L(2, R)$ theory (whose energy momentum tensor has a term proportional to $\partial J_{3}$ ) with $J^{-}$, having spin zero, set to a constant equal to

2 This is not necessarily a desirable thing to do for $k>0$, which is like a negative level for $S U(2)$. 
$\sqrt{\mu}$ [38]. This gauging introduces a pair of ghosts $B, C$ of spins 1,0 with a KPZ reduction operator given by

$$
Q_{K P Z}=B\left(J^{-}-\sqrt{\mu}\right)
$$

In addition to this, one adds the matter system and the diffeomorphism $b, c$ ghosts. One then considers, on the reduced Hilbert space, the usual string BRST operator

$$
Q_{B R S T}=c\left(T_{m}+T_{l}+\frac{1}{2} T_{\text {ghost }}\right)
$$

where the subscript $m$ refers to matter and $l$ refers to the combined twisted $S L(2, R)$ and $B, C$ system. The level of $S L(2, R)$ that one needs is fixed by the central charge of the matter [20], and in the case of matter with $c=1$ it is given by $k=3$. Moreover, as pointed out in [39], it turns out that in the KPZ formulation we have to use a Wakimoto representation for $S L(2, R)$ in order to get agreement with the usual $c=1$ theory. So the full Hilbert space in the KPZ description for $c=1$ matter represented by a scalar field $X$ is given by

$$
\mathcal{H}=[\phi]+[\beta, \gamma]+[B, C]+[X]+[b, c]
$$

which is exactly the same Hilbert space we had in the KS case discussed in the previous section, with the same expressions for the energy momentum tensors (in both cases the $S L(2, R)$ is twisted and at level 3$)$.

However, the BRST structure seems at first sight to be different: In the KPZ case we first impose the constraint corresponding to the BRST charge

$$
Q_{K P Z}=B(\beta-\sqrt{\mu})
$$

This means that the $B, C, \beta, \gamma$ degrees of freedom can be ignored (after setting $\beta=\sqrt{\mu}$ ), and we are left with $[\phi]+[X]+[b, c]$ on which the physical subspace is obtained by considering the string BRST $Q_{B R S T}$. This gives the usual DDK-type expressions for the physical states in terms of $X$ and $\phi$. In the KS theory we have instead to reduce the Hilbert space first under

$$
Q_{U(1)}=C\left(\beta \gamma-c b-\partial X^{-}\right)
$$

and then impose the Kazama-Suzuki BRST

$$
Q^{+}=c J^{+}=c\left(\beta \gamma^{2}-\sqrt{2} \gamma \partial \phi+3 \partial \gamma\right)
$$


as we did in the previous section. As discussed there, the cohomologies are represented by the same expressions as in $c=1$ theory, with some extra $\beta$ dependence which disappeared when we set $\beta=\sqrt{\mu}$. In other words, the cohomology states of the KPZ theory can be chosen, by modifying with $Q_{K P Z}$ trivial fields, to have these extra $\beta$ dependences and so can be chosen to coincide with those of KS theory. It is desirable to explain this coincidence at the level of the BRST operators. The following shows this directly.

Note that in both the KPZ formulation and in the KS model a twisted $N=2$ algebra is realized. The two supercharges of the algebra in the KPZ case are $Q_{B R S T}$ and $b_{0}$ and in the KS case are $Q^{+}$and $Q^{-}$. Moreover, we have

$$
\left\{Q_{B R S T}, b_{0}\right\}=L_{0}
$$

and

$$
\left\{Q^{+}, Q^{-}\right\}=L_{0}+\left\{Q_{U(1)}, \Lambda\right\}
$$

where the last equality is just the statement of the twisted $N=2$ symmetry of the KS model. This symmetry is valid modulo the $U(1)$ constraint, explaining the existence of the second term for some $\Lambda$. Now $Q^{-}$differs from $b$ only because of an extra $\beta$, so using the $\mathrm{KPZ}$ constraint we see that it is equivalent to $b_{0}$.

Consider now the subspace of the Hilbert space where the KPZ constraint and the KS constraint are both satisfied. This is compatible in that if we allow both positive and negative powers of $\beta$, any state in the Hilbert space can be made to satisfy the KS constraint by dressing with powers of $\beta$ without affecting the KPZ constraint, as $\beta$ is set to a constant. On this reduced Hilbert space we see that the two BRST charges $Q_{B R S T}$ and $Q^{+}$, which are defined by the nilpotency condition and the anti-commutation relations above, are equivalent, since their defining relations are equivalent.

A more direct way of showing the equivalence of $Q_{B R S T}$ and $Q^{+}$, which was pointed out to us by V. Sadov (following a similar computation done in [40]), is to note that

$$
\beta^{-1} Q_{B R S T}=Q^{+}+\left\{Q_{U(1)}, \oint c B\left(\gamma-\beta^{-1} \partial X^{+}\right)+c \beta^{-1} \partial B\right\}
$$

Thus the two BRST charges are equivalent upto a $Q_{U(1)}$-exact term.

To complete the proof that the (twisted) KS theory (coupled to topological gravity) and the KPZ theory are really the same, we have to show that the rules for computations of the correlation functions are also the same. As far as the screening operators are concerned, they are the same for both KPZ and KS because they come from the same 
underlying $S L(2, R)$ theory. The only other thing we need to check is the fact that in the KPZ case, as in all string theories, we have to make $3 g-3$ insertions of $b$ ghosts (and similarly for the right-movers). In the context of twisted KS theory coupled to topological gravity, this role is played by $3 g-3$ insertions of $G^{-}=b \beta$. This is the same as in KPZ when we use our freedom in the KPZ theory to make $Q_{K P Z}$ trivial modification to $b$. We have thus shown that the twisted KS theory discussed in the previous section, coupled to topological gravity, is equivalent to the $c=1$ theory coupled to gravity in the KPZ formulation.

\section{Partition Function Computation}

In order to understand whether the above correspondence between the KS theory and non-critical $c=1$ coupled to gravity is practically useful or simply an elegant reformulation, we need to ask whether in the new formulation the theory is amenable to explicit computation. Remarkably the answer to this question is in the affirmative and clearly demonstrates the power of topological methods in this reformulation. In fact, this particular KS theory coupled to topological gravity has been considered by Witten [17] who showed how to compute the partition function at arbitrary genus, and certain correlation functions.

In [17] it was found that if the level $k$ of the $S U(2)$ algebra is continued to -3 , the partition function of the twisted KS theory at arbitrary genus (which is nonzero without any need for field insertions because $\hat{c}=3$ ) is given by the Euler characteristic of the moduli space of Riemann surfaces (This can be computed using a matrix model due to Penner [16].) On the other hand, it had already been discovered [15] that the partition function of the $c=1$ matrix model at the self-dual radius, is given at arbitrary genus by the Euler characteristic of the moduli space of Riemann surfaces. The reason for this correspondence between the partition function of the KS model and that of the $c=1$ matrix model at self-dual radius was not understood, and was one of our main motivations for undertaking this work. This is now explained by our proof that the $c=1$ non-critical string is equivalent to the special KS model. Thus we have demonstrated that the matrix model and continuum results for the partition function of non-critical $c=1$ matter at the self-dual radius are the same!

The Lagrangian formulation of the KS model, which is most suitable for making use of topological field theory techniques in computing correlations functions, has been discussed 
in [17], to which we refer the reader for more details. Here we quote some of the main facts relevant to our problem. A subset of chiral fields, i.e., $Q^{+}$invariant fields, which exists for all $k$, was found to be concentrated on $S U(2)$ group configurations $g$ for which

$$
g=\left(\begin{array}{cc}
e^{i \theta} & 0 \\
0 & e^{-i \theta}
\end{array}\right)
$$

The chiral fields are represented by powers of $g_{11}$. Let $U_{r}=g_{11}^{r}$, and let us allow $r$ to be both positive and negative 3 . We will discuss below how these fields are related to the tachyon fields discussed in section 3 . The result for the $n$-point function of these operators at arbitrary genus is given by [17]

$$
\left\langle U_{r_{1}} U_{r_{2}} \ldots U_{r_{s}}\right\rangle_{g}=\int_{\overline{\mathcal{M}}_{g, s}} c_{T}(\nu)
$$

where $\overline{\mathcal{M}}_{g, s}$ denotes the compactified moduli space of Riemann surfaces of genus $g$ with $s$ punctures, $\nu$ is a bundle over this space whose fibers are given by

$$
V=H^{0}\left(\Sigma, K^{\otimes 2} \otimes_{i} O\left(z_{i}\right)^{-r_{i}}\right)
$$

where $\Sigma$ is a Riemann surface of genus $g$ and $K$ is its canonical line bundle, and $c_{T}$ in (5.2) denotes the top chern class of the bundle. With no $U$ 's inserted, (5.2) gives the Euler characteristic of $\overline{\mathcal{M}}_{g}$.

To understand the significance of the $U_{r}$ fields, note that $g_{11}$ is a HWS (because it is the 11 component of the matrix field). In fact, $g_{11}^{r}$ will belong to the highest weight state of the representation $D_{r / 2}^{-}$, which should thus correspond to the tachyon states $V_{r / 2}$ defined in Eq.(3.17). In particular, as discussed there, $V_{-1 / 2}$, (which comes from $r=-1$ ) corresponds to the cosmological constant operator. Using (5.2) we can compute the s-point function of the cosmological constant operator because in that case the Euler character of $\nu$ with $V=H^{0}\left(\Sigma, K^{\otimes 2} \otimes_{i} O\left(z_{i}\right)\right)$ is the same as the Euler character of $\overline{\mathcal{M}}_{g, s}$. From [15] this is known to be the same answer as that of the matrix model with $s$ insertions of the cosmological constant operator. So we find further confirmation that the continuum and matrix model results match.

There is a conservation law in (5.2) which implies [17]

$$
\sum_{i}\left(r_{i}+1\right)=0
$$

3 The choice of negative powers of $g_{11}$ is more natural if we consider the group to be $S U(1,1)$ (since the level is negative in $S U(2)$ language) as in this group, $g_{11}$ is never zero. 
This conservation law is independent of the genus $g$. In fact it is exactly what we would expect given the identification of $U_{r}$ with a HWS with $j=r / 2$, because we have from the identification of section 3 , that the $X$-momentum $k$ (up to a factor of $1 / \sqrt{2}$ ) is given by (3.17):

$$
k=2 j+1=r+1
$$

Therefore (5.3) is equivalent to

$$
\sum_{i} k_{i}=0
$$

This is just matter momentum conservation, which holds in every genus.

Let us now turn to the computation of the 2,3 and 4 point functions of the tachyon operators $U_{r}$ on the sphere. These computations have also been performed in [17] (all we need to do is make a simple modification in the case of 4 point functions on the sphere): To compute the two point function, it is more natural to first compute the three point function, and then set one of the operators to be the cosmological constant operator. The three-point function on the sphere is (eq. 3.36 of [17])

$$
\left\langle U_{r_{1}} U_{r_{2}} U_{r_{3}}\right\rangle_{g=0}=\delta_{\left(r_{1}+r_{2}+r_{3}+3\right)}
$$

which using (5.4) is simply the statement that the three point function is 1 if the matter momentum conservation is satisfied and zero otherwise. To obtain the two point function, we simply need to set one of the $r_{i}$ to -1 , which again implies that the two point function is 1 if the two momenta are equal and opposite. (We are taking the cosmological constant to be equal to 1 , but the right powers of $\mu$ can always be restored by considering the amount of momentum violation for the $\phi$ field).

Now we turn to 4-point tachyon correlators on the sphere. The four point computation in the $\mathrm{KS}$ model has been done for positive $S U(2)$ level in [17]. This can be adapted to our case by a slight modification in Eq.(4.62) of that paper (with $\gamma=-1$ ):

$$
\left\langle U_{r_{1}} U_{r_{2}} U_{r_{3}} U_{r_{4}}\right\rangle=\left[\frac{-1}{2} \max \left(r_{1}+r_{2}, r_{3}+r_{4}\right)-\frac{2}{3}\right]+\left(r_{2} \leftrightarrow r_{3}\right)+\left(r_{2} \leftrightarrow r_{4}\right) .
$$

Using $k=r+1$ and $\sum k_{i}=0$ this is equal to

$$
\left\langle U_{r_{1}} U_{r_{2}} U_{r_{3}} U_{r_{4}}\right\rangle=-\frac{1}{2}\left|k_{1}+k_{2}\right|-\frac{1}{2}\left|k_{1}+k_{3}\right|-\frac{1}{2}\left|k_{1}+k_{4}\right|+1
$$

4 This result is also valid when one considers rational radii by going to the $n$-fold cover of $S U(1,1)$, and agrees with the standard results of $c=1$ theory. 
which is equal to the four point tachyon amplitude on genus zero computed using either the matrix model [41] or the DDK approach [7] (with the leg factors absorbed into the definition of $U_{r}$ ).

We have thus found that the partition function to all genus, the $n$-point function of the cosmological constant to all genus, and the $n$-point function of tachyons up to $n=4$ at genus zero, can all be explicitly computed in the KS model, and agree with the corresponding matrix model results.

Since we know explicitly the $n$-point tachyon correlators in the matrix model [41] [5] and there is an expression (given in Eq.(5.2) ) for the corresponding amplitudes in the KS theory, we have a conjectured equality between these quantities to all genus and for all $n$.

We have been somewhat imprecise as to which chirality tachyon amplitude we are considering as there is a puzzle here. From the naive relation we discussed above for the relation of $U_{r}$ with the HWS tachyon state with $j=r / 2$, the two and three point function computation seem to be in contradiction with the computation using explicit representations we obtained in section 3. In particular two HWS tachyon fields do not have a non-vanishing two point function. We need the dual field which corresponds to a tachyon of opposite chirality which includes a factor $\beta^{-1}$ in order to give a non-zero answer. The same is true for the three point functions.

We believe that the clue to resolving this puzzle is to note the role of the screening current (3.30). Indeed this suggests that $g_{11}^{r}$ for $r>0$ is to be identified with the dual tachyon fields which have extra factors of $\beta^{-1}$ and are of opposite chirality. The way the two point function comes out to be non-vanishing in this case is by using the screening current (3.30) to take care of $\phi$ momentum conservation. Indeed, application of an appropriate number of (3.30) currents to tachyons of one chirality seems to map them to tachyons of the other chirality, by replacing a negative Liouville dressing with a positive one. This is expected in view of the fact that we are at a non-zero cosmological constant and the Liouville momentum conservation is not valid, or more precisely it is valid only when we also count the factors of the cosmological constant. Therefore, the fact that only one set of tachyons appears in (5.2) should be related to the fact that for each matter momentum there is a unique state with negative Liouville dressing which should be identified with $U_{r}$.

As we know, the $c=1$ model has, in addition to tachyon states, an infinite number of discrete states. As we discussed in section 3, they also appear in the KS theory. How do we compute correlation functions involving some discrete states using topological techniques? This is a very important question to which an answer needs to be found. There is also 
a complementary question: We know that for each tachyon field $U_{r}$ there is an infinite series of gravitational descendants $\tau_{n}\left(U_{r}\right)$ for which the abstract definition of correlation function, a generalization of (5.2) is given in [17]:

$$
\left\langle\tau_{n_{1}}\left(U_{r_{1}}\right) \tau_{n_{2}}\left(U_{r_{2}}\right) \ldots \tau_{n_{s}}\left(U_{r_{s}}\right)\right\rangle=\int_{\overline{\mathcal{M}}_{g, s}} c_{T}(\nu) \cdot \prod_{i=1}^{s}\left(c_{1}\left(\mathcal{L}_{(i)}\right)\right)^{n_{i}}
$$

where $c_{1}\left(\mathcal{L}_{(i)}\right)$ denotes the first chern class of the line bundle on moduli space whose fiber is the space of one-forms at the puncture on the Riemann surface. The selection rule now gets modified to

$$
-\sum_{i}\left(r_{i}+1\right)+\sum_{i} n_{i}=0
$$

One is tempted to identify the extra states of the topological theory (the gravitational descendants) with the missing states of KS theory (the discrete states). It seems that the most natural description of the topological descendants may involve using the dual Wakimoto module discussed in section 3, where the topological gravity multiplet is automatically available. This does not appear to be completely straightforward, however, and this problem is under investigation.

One may also ask if one can use topological techniques to compute correlation function of the $c=1$ theory away from the self-dual point. In principle this must be possible because following the logic of section 3 , we see that going to the $n$-fold cover of $S U(1,1)$, the $X-$ field will live on a circle whose radius is $n$ times the self-dual radius. It should be possible to translate this geometric statement into a computation of the partition function 5 by carefully finding the modifications needed in [17] when one goes to the multiple cover.

\section{Black Hole Interpretation}

We have seen in the previous sections that $c=1$ non-critical string is equivalent to a superconformal $S L(2, R) / U(1)$ coset. Let us compare this with a proposal in [13]

5 One might formally think that this should give as partition function the Euler characteristic of the moduli space of Riemann surfaces with a $Z_{n}$ flat connection, but this only modifies the answer from the self-dual result by the factor $n^{g}$ at genus $g$, whereas the expected answer from the $c=1$ matrix model is a polynomial in $n$ and $n^{-1}$, invariant under $n \leftrightarrow n^{-1}$ with the highest power $n^{g}$. 
where it was suggested that non-critical $c=1$ string may be related to an $S L(2, R) / U(1)$ background.

In fact, the construction of section 3 is very reminiscent of similar considerations in the context of the black hole of [13] done in [42]. In that case, with the choice of level $k=9 / 4$, the central charge of the $S L(2, R)$ is 27 . Taking a $U(1)$ coset decreases the central charge by 1 to give 26 and then the ghost system $b, c$ with central charge -26 is added, to get total central charge zero:

$$
\frac{\left.S L(2, R)\right|_{27}}{U(1)} \otimes[b, c]
$$

Our supersymmetric version, on the other hand, can be described in the following way: we start with an $S L(2, R)$ at level $k=3$ with twisted central charge 27 (twisted in the sense that we have added a term proportional to $\partial J_{3}$ to the energy momentum tensor), add to it a $b, c$ system with central charge -26 , giving a net central charge of 1 , and then take a $U(1)$ quotient which decreases the central charge by 1, to give zero net central charge:

$$
\frac{\left.S L(2, R)\right|_{27} \otimes[b, c]}{U(1)}
$$

There are two differences between these two theories. One is that the supersymmetric model that we study contains a twisted $S L(2, R)$ theory, which is why the two theories have the same central charge 27 at two different levels $k=9 / 4$ and $k=3$. The second is that in taking the $U(1)$ coset in the case of (6.1) there is no mixing with the $[b, c]$ system, whereas for us, the $U(1)$ in (6.2) mixes the 'matter' and the 'ghost' system, as its generator is given by $J_{3}+b c$.

The question is, which background does our theory (given by (6.2)) describe? In fact this has already been studied in Refs. 43] [44] where it is concluded that the supersymmetric coset also describes the same black hole configuration! So we are led to a puzzle: If we are interested in describing bosonic strings propagating in a black hole background, which coset is the correct choice: 6.1) or (6.2)?

Based on experience with static string solutions, one might be inclined to pick (6.1) as the correct choice, as the ghost and matter systems are always treated independently, whereas (6.2) mixes the two. However, one expects that for time dependent backgrounds there should be a mixing between the matter and the ghosts. There are various ways to see this. Perhaps the simplest indication that this might happen is in the proof of the noghost theorem for bosonic strings given in [45]. It is crucial in their proof that the signature 
character of the ghost Hilbert space cancels part of the matter signature character given by an uncompactified flat $1+1$ dimensional spacetime. So if we change the matter theory such that there is no 1+1 dimensional uncompactified flat spacetime left, then there is bound to be some change in our ghost system as well, to maintain the no-ghost theorem. Therefore for the $1+1$ dimensional black hole geometry one expects a mixing, and indeed (6.2) is a very "mild" mixing of the matter system with the ghost system, via a $U(1)$ constraint. The fact that the matrix model partition function agrees to all genus with the partition function of (6.2) is, in our opinion, a strong indication that the correct theory describing bosonic string propagation in the black hole background is in fact (6.2) and not (6.1).

This also suggests that maybe the time dependent backgrounds are described in bosonic string theory by a twisted $N=2 \mathrm{SCFT}$ as is the case here. This is a very interesting question for further research, even in the context of critical strings.

Given this connection between matrix models and black holes, it is natural to ask what we may learn about black hole singularity. So far we have been talking about the Euclidean black hole. The Minkowski version is obtained by changing the $U(1)$ imbedding [13]. In our case, which has an $N=2$ supersymmetry, there is an observation in [44 that the simplest chiral fields (those given by $g_{11}^{r}$ ) correspond to the points on the black hole singularity! This is very significant as it implies that the singularity itself encodes all the physical degrees of the freedom of the black hole and that it is a topological degree of freedom, a notion which was previously suggested in [13]. Perturbing the theory by throwing in tachyons is equivalent to perturbing the singularity structure, namely, changing the topological $N=2$ theory by topologically relevant perturbations.

Another question to ask is whether string theory makes the singularity go away, or more precisely what is the fate of the singularity. This is a more difficult question to answer. In order to discuss this point let us first consider the similar but simpler situation of orbifold singularities [46]. In that case also, the singularities can be viewed as topological degrees of freedom of the $N=2$ theory, as was discussed in [47](see also 448]). In fact the orbifold singularities contribute to Witten's index $\operatorname{Tr}(-1)^{F}$. In the case of orbifolds it is well known that singularities are harmless for string propagation 49] and one does not need to get rid of them to make the theory consistent, even though if we were describing point particle propagation in the presence of orbifolds we would get an inconsistent theory. For strings not only it is not needed to get rid of the singularity, but in fact any finite perturbation of the theory will leave a trace of the singularity, because $\operatorname{Tr}(-1)^{F}$, being an 
index, cannot change. Perturbation will resolve the singularity but will still lead to the same contribution to Witten index.

The above situation is very similar to that of black holes. In the case of black holes also, the points of singularity contribute to $\operatorname{Tr}(-1)^{F}$, as they are topological. Moreover being described by a conformal coset theory suggests that the strings have a non-singular propagation on the black holel. To see the fate of the black hole, we have to consider string loop corrections in the Minkowskian version. As we discussed, the matrix model is equivalent to the Euclidean black hole and it is thus not clear how the computations in the Euclidean set up will have to be interpreted for the Minkowskian version. But since the black hole singularity is topological, whatever happens to the singularity it cannot quite disappear, because it contributes to a supersymmetric index!

The Minkowskian version of the Kazama-Suzuki model will have to be studied in more detail to shed light on aspects of the black hole. It is amusing that even though the matrix model is equivalent to Euclidean black hole, it involves 'scattering' for tachyons. This is because one allows exponentially growing (or decaying) solutions in one of the Euclidean directions. It would be interesting to connect the scattering matrix thus obtained to that for the Minkowskian black hole.

At any rate, it is quite remarkable that with the dictionary we have found, the partition function of the Euclidean 2d black hole can be computed for all genus, and is given by the Euler characteristic of the moduli space of Riemann surfaces of that genus.

\section{Generalizations and Conclusions}

We have demonstrated that $c=1$ matter coupled to gravity with non-zero cosmological constant is equivalent to a topological superconformal $S L(2, R) / U(1)$ coset coupled to topological gravity. This solves the problem of finding a topological field theory for the $c=1$ string, and leads to a systematic computation of the continuum theory correlations using topological techniques. All the results that have been checked (including correlation of arbitrary number of cosmological constant operators at all genus and up to four-point tachyon scattering amplitude on genus zero) agree with those of the $c=1$ matrix model. Moreover, we have shown how this theory is related to a bosonic string propagating in a two dimensional black hole background.

6 This needs to be verified by considering correlations of the Minkowskian coset theory. 
There are a number of directions that are suggested by these observations: We now have an exact, completely solvable representation of the Euclidean black hole in terms of the $c=1$ matrix model. What does this teach us about black holes? How can we use similar ideas to describe other time-dependent string solutions? We have discussed some aspects of these questions in section 6 .

Another question worth investigating is to show explicitly that the results of the matrix models tachyon scattering amplitudes for all genus given in [41] agree with that predicted by this correspondence and given by (5.2). Turning things around, we thus have a Kontsevich-like representation of the invariants (5.2) given by a Penner-like matrix integral as obtained in [5].

Finally, it is natural to ask if the above ideas can be generalized to other models with $c<1$. The most obvious generalization would be just to change the level of the KS coset model. Even though this has been used in conjunction with topological gravity beginning with the work of $\mathrm{Li}$ [50], it is not in the same spirit as what we are doing here in that the ghost system is part of the matter system for us and we do not have to add it by hand. In particular as is clear from (3.5), only for $k=3$ do we get the $b, c$ ghost system with the correct spins.

Instead it turns out that a remark by Frenkel (see appendix A), that the superconformal KS cohomology computation at $k=3$ is equivalent to the bosonic $S L(2, R) / S L(2, R)$ at the same level, can be used to generalize the above construction [21]. Indeed it was already known that the physical states of $S L(2, R) / S L(2, R)$ topological theory are isomorphic to those of matter coupled to gravity [51] 40]. However there are two new ingredients here which make this work: first, one should take a twisted bosonic $S L(2, R) / S L(2, R)$ theory, and secondly it turns out that by a careful reorganization of the BRST, one obtains an $N=2$ superconformal algebra for this theory [21]. The twisting has the effect of making one pair of the gauge ghosts act as the $b, c$ system with the correct spin, and the other two pairs act as KPZ reduction [20] and Hamiltonian reduction [52] of the rest of the degrees of freedom (to Liouville and matter respectively). The possible connections of this twisted $S L(2, R) / S L(2, R)$ theory to three dimensional gravity are also currently under investigation [21].

We would like to thank M. Bershadsky, D. Ghoshal, C. Imbimbo, D. Jatkar, S. Mathur, 
G. Moore, H. Ooguri, V. Sadov, E. Verlinde, H. Verlinde and E. Witten for valuable discussions. We are greatly indebted to E. Frenkel for taking up the question of computation of the cohomology of the KS coset model presented in appendix A.

S.M. would like to acknowledge the kind hospitality of the relevant departments at Harvard University, Princeton University, Ecole Normale Superieure, and INFN, Genova, where part of this work was done.

The research of C.V. was supported in part by Packard Foundation and NSF grants PHY-89-57162 and PHY-87-14654.

\section{Appendix A. Cohomology of Kazama-Suzuki Model}

\section{by Edward Frenkel}

In this Appendix we will compute the spins of the cohomology states of the KazamaSuzuki (KS) model (cf. Section 3).

For a module $M$ over the affine Kac-Moody algebra $\hat{S L}(2)$ of level -3 let us consider the complex $M \otimes[X] \otimes[b, c] \otimes[C, B]$, graded by the ghost number, with respect to the action of the differential

$$
\int\left[C\left(J^{3}-i \frac{\partial X}{\sqrt{2}}\right)+c J^{+}-C c b\right] d z .
$$

Let us denote by $H^{q}(M)$ the space of cohomology states of this complex (relative to the 0th mode of $C$ ), of ghost number $q$. If $M$ is the Wakimoto module $W_{j}$ with HWS $D_{j}^{-}$, with $j$ integral or half-integral, then $H^{q}\left(W_{j}\right)$ is exactly the space of states of ghost number $q$ of the KS model, of ghost number $q$. We will write $H^{q}(M)=\left\{j_{1}, \ldots, j_{n}\right\}$, if $H^{q}(M)$ is linearly spanned by states of spins $j_{1}, \ldots, j_{n}$, and will use the notation $S_{j_{1}, j_{2}}$ for the set $\left\{j_{1}, j_{1}-1, \ldots, j_{2}+1, j_{2}\right\}$.

Theorem (a) If $j=-1 / 2$, or $j=-1$, then $H^{1}\left(W_{j}\right)=\{j\}$;

(b) If $j \geq 0$, then $H^{1}\left(W_{j}\right)=S_{j,-j-1}, H^{2}\left(W_{j}\right)=S_{j-1,-j-1}$;

(c) If $j<-1$, then $H^{1}\left(W_{j}\right)=S_{-j-2, j}, H^{0}\left(W_{j}\right)=S_{-j-2, j+1}$;

and all other $H^{q}\left(W_{j}\right)=0$.

To prove this Theorem, we will use the following result on the structure of the Wakimoto modules with negative levels, which was proved in [30] (where we used the opposite notations for Wakimoto and dual Wakimoto modules): for $j \geq-1 / 2$ the module $W_{j}$ is 
isomorphic to the Verma module with the same HWS, and for $j<-1 / 2$ the module $W_{j}^{*}$ is isomorphic to the Verma module with the same HWS.

This result is quite surprising. Indeed, the Verma modules are defined as the modules, freely generated from their HWS under the action of the subalgebra of $\hat{S L}(2)$, spanned by $J_{n}^{3}, n<0, J_{n}^{+}, n<0$, and $J_{n}^{-}, n \neq 0$, whereas the Wakimoto modules $W_{j}$ (or dual Wakimoto modules $\left.W_{j}^{*}\right)$ are defined by explicit embeddings of $\hat{S L}(2)$ into the free fields. These embeddings are such that the modules $W_{j}\left(W_{j}^{*}\right)$ are free over the Lie subalgebra, spanned by $J_{n}^{3}, n<0$ and $J_{n}^{-}, n \leq 0\left(J_{n}^{3}, n<0\right.$ and $\left.J_{n}^{+}, n<0\right)$, and co-free over the Lie subalgebra, spanned by $J_{n}^{-}, n>0\left(J_{n}^{+}, n \geq 0\right)$ (cf. [53]).

Now, the structure of Verma modules over $\hat{S L}(2)$ is known from [54, and this allows us to describe the structure of $W_{j}$ and $W_{j}^{*}$.

Our computation is based on the following result.

Lemma For the dual Wakimoto module $W_{j}^{*}$ we have: $H^{1}\left(W_{j}^{*}\right)=\{j\}$, and $H^{q}\left(W_{j}^{*}\right)=0$ for $q \neq 1$.

This follows from the properties of $W_{j}^{*}$ with respect to the action of the operators $J_{n}^{3}$ and $J_{n}^{+}$, described above.

The modules $W_{-1}$ and $W_{-1 / 2}$ are irreducible and therefore they are isomorphic to $W_{j}^{*}$. Hence, part (a) of the Theorem follows from the Lemma. We can prove the rest of the Theorem by induction, separately for integral and half-integral values of $j$.

We will demonstrate it here in the case of integral spins. The case of half-integral spins can be pursued along the same lines.

The modules $W_{j}$ with $j \geq 0$ are isomorphic to Verma modules and have the structure:

$$
\bullet^{I_{j}} \longrightarrow \bullet^{I_{-j-1}} \longrightarrow \bullet^{I_{j-1}} \longrightarrow \ldots \bullet^{I_{0}} \longrightarrow \bullet^{I_{-1}} \text {. }
$$

The modules $W_{j}$ with $j<0$ are isomorphic to dual Verma modules and have the structure:

$$
\bullet^{I_{j}} \longleftarrow \bullet^{I_{-j-2}} \longleftarrow \bullet^{I_{j+1}} \longleftarrow \ldots \bullet^{I_{0}} \longleftarrow \bullet^{I_{-1}}
$$

Here $I_{j}$ denotes the irreducible module with HWS $D_{j}^{-}$. These diagrams have the following meaning: the dots represent the irreducible subfactors of $W_{j}$, and the arrows show, which way we can get from one of them to another.

The diagram for the module $W_{j}^{*}$ can be obtained from the diagram for the module $W_{j}$ by reversing the arrows. 
Assume that we have already proved the Theorem for all integral values of $j$, such that $|j+1| \leq n$, where $n \geq 0$ is an integer.

In particular, the diagram (A.2) with $j=-n-1$ and the reverse to the diagram (A.1) with $j=n$ imply that $I_{n}$ is a submodule of $W_{n}^{*}$, and the quotient is isomorphic to $W_{-n-1}$. Therefore, the following exact sequence holds:

$$
0 \rightarrow I_{n} \rightarrow W_{n}^{*} \rightarrow W_{-n-1} \rightarrow 0
$$

Recall that every exact sequence of modules $0 \rightarrow A \rightarrow B \rightarrow C \rightarrow 0$ leads to the following exact sequence of cohomologies

$$
\ldots \rightarrow H^{q}(A) \rightarrow H^{q}(B) \rightarrow H^{q}(C) \rightarrow H^{q+1}(A) \rightarrow \ldots
$$

Our sequence (A.3) gives:

$$
0 \rightarrow H^{0}\left(W_{-n-1}\right) \rightarrow H^{1}\left(I_{n}\right) \rightarrow H^{1}\left(W_{n}^{*}\right) \rightarrow H^{1}\left(W_{-n-1}\right) \rightarrow H^{2}\left(I_{n}\right) \rightarrow 0
$$

because all other cohomologies are 0. By our induction assumption and the Lemma, we have: $H^{1}\left(W_{n}^{*}\right)=\{n\}, H^{1}\left(W_{-n-1}\right)=S_{n-1,-n-1}$. Therefore, there can be no non-trivial map between them, and the sequence (A.4) splits into two:

$$
0 \rightarrow H^{0}\left(W_{-n-1}\right) \rightarrow H^{1}\left(I_{n}\right) \rightarrow H^{1}\left(W_{n}^{*}\right) \rightarrow 0
$$

and

$$
0 \rightarrow H^{1}\left(W_{-n-1}\right) \rightarrow H^{2}\left(I_{n}\right) \rightarrow 0
$$

According to (A.5), $H^{1}\left(I_{n}\right)=H^{0}\left(W_{-n-1}\right)+H^{1}\left(W_{n}^{*}\right)=S_{n,-n}$, and according to (A.6), $H^{2}\left(I_{n}\right)=H^{1}\left(W_{-n-1}\right)=S_{n-1,-n-1}$, and also $H^{q}\left(I_{n}\right)=0$ for $q \neq 1,2$.

Further, we have the exact sequence

$$
0 \rightarrow W_{-n-1}^{*} \rightarrow W_{n} \rightarrow I_{n} \rightarrow 0
$$

By the same method, we obtain $H^{1}\left(W_{n}\right)=H^{1}\left(W_{-n-1}^{*}\right)+H^{1}\left(I_{n}\right)=S_{n,-n-1}, H^{2}\left(W_{n}\right)=$ $H^{2}\left(I_{n}\right)=S_{n-1,-n-1}$, and $H^{q}\left(W_{n}\right)=0$ for $q \neq 1,2$.

Now, using this result and the exact sequence

$$
0 \rightarrow W_{n} \rightarrow W_{-n-2}^{*} \rightarrow I_{-n-2} \rightarrow 0
$$


we obtain in the same fashion: $H^{1}\left(I_{-n-2}\right)=S_{n-1,-n-2}, H^{0}\left(I_{-n-2}\right)=S_{n,-n-1}$, and $H^{q}\left(I_{-n-2}\right)=0$ for $q \neq 0,1$. This, together with the exact sequence

$$
0 \rightarrow I_{n} \rightarrow W_{-n-2} \rightarrow W_{n}^{*} \rightarrow 0
$$

gives: $H^{1}\left(W_{-n-2}\right)=S_{n,-n-2}, H^{0}\left(W_{-n-2}\right)=S_{n,-n-1}$, and $H^{q}\left(W_{-n-2}\right)=0$ for $q \neq 0,1$.

Thus, we have now proved our statement for integral values of $j$, such that $|j+1| \leq$ $n+1$. This completes the proof of the Theorem.

\section{Remarks}

(1) In the course of proving our Theorem, we have computed the cohomology $H^{q}\left(I_{j}\right)$, corresponding to the irreducible representations $I_{j}$. This cohomology is the same as that of $W_{j}$, except that one state is missing in $H^{1}\left(I_{j}\right)$ : of spin $-j-1$ for $j \geq 0$, and of spin $-j-2$ for $j \leq-1$.

(2) It is known that $W_{j}$ is irreducible, if $j$ not integral or half-integral. In that case $W_{j}$ is isomorphic to $W_{j}^{*}$, and therefore the only cohomology class, which occurs, is the HWS.

(3) The technique that we have described will work for other negative rational levels as well. We can again use the fact that $W_{j}$ are isomorphic to Verma modules for $j \geq-1 / 2$, and $W_{j}^{*}$ are isomorphic to Verma modules for $j<-1 / 2$ [30]. However, in general, the structure of Verma modules can be more complicated: we may have not one, but two strings of singular vectors (cf. [54], [30]).

(4) We would like to remark that at negative rational levels there are no good projections from Wakimoto modules to irreducible modules, in contrast to irrational, or positive rational levels.

For irrational levels, the irreducible representation of integral or half-integral spin $j \geq 0$ can be defined as the 0th homology of the complex

$$
0 \leftarrow W_{j} \leftarrow W_{-j-1} \leftarrow 0
$$

or as the 0th cohomology of the complex

$$
0 \rightarrow W_{j}^{*} \rightarrow W_{-j-1}^{*} \rightarrow 0
$$

In these complexes the only non-trivial differential is a multiple integral of the $(2 j+1)$ st power of the corresponding screening current (cf. formula (3.30)). 
For positive rational levels, we should extend the complexes (A.7) and (A.8) to a twosided resolution as explained in [53, [55]. The resulting complexes will have the property that all but the middle cohomology vanishes, and the middle cohomology is isomorphic to the irreducible representation.

At rational negative levels (for example, $k=-3$ ) the complexes (A.7) and (A.8) no longer work, because the power of the screening charge is equal to 0 in this case. On the other hand, the complex (A.3) that we used in the proof of our Theorem, includes both Wakimoto and dual Wakimoto modules, and therefore the corresponding differential can not be expressed in a local form.

We may also consider the following complex

$$
0 \leftarrow W_{j} \leftarrow W_{j-1} \leftarrow 0,
$$

(for $j \geq 0$ ) with the screening charge (3.30) as the differential. However, the analysis of the structure of the Wakimoto modules, given by the diagram (A.1), shows that the 0th homology of this complex is not the irreducible module, but a module $X_{j}$, which has the following structure:

$$
\bullet^{I_{j}} \longrightarrow \bullet^{I_{-j-1}} \text {. }
$$

It contains two irreducible factors: $I_{j}$ and $I_{-j-1}$. Of course, we can introduce a resolution of $I_{j}$, consisting of the modules $X_{n}$, but it can not be made into a resolution, which consists of the Wakimoto modules.

(5) Finally, let us remark that the cohomology states of KS model at $k=-3$ are in one to one correspondence with the cohomology states of bosonic $S L(2) / S L(2)$ theory at level -1 . Indeed, let us consider the complex of (relative) BRST cohomology of the whole algebra $S L(2)$ with coefficients in $W_{j} \otimes W_{j^{\prime}}$, where $W_{j^{\prime}}$ is the Wakimoto module of level -1 with HWS of spin $j^{\prime}$ (cf. [51]). Then, by the properties of the Wakimoto modules with respect to the action of the operators $J_{n}^{-}$, described above, the cohomology of this complex reduces to spin $-j^{\prime}-1$ sector of the cohomology of KS model. Indeed, we can strip off the bosonic ghosts, involved in the module $W_{j^{\prime}}$, together with the fermionic ghosts, corresponding to the current $J^{-}$, without changing the cohomology.

The cohomology classes of KS model, constructed in Section 3, can then be viewed as symbols of the cohomology classes of the $S L(2) / S L(2)$ model with respect to a certain filtration. 


\section{References}

[1] P. Ginsparg and G. Moore, Proceedings of TASI 92, ed. J. Harvey and J. Polchinski.

[2] E. Brezin, V. Kazakov and Al.B. Zamolodchikov, Nucl. Phys. B338 (1990) 673;

D. Gross and N. Miljkovic, Phys. Lett. 238B (1990) 217;

P. Ginsparg and J. Zinn-Justin, Phys. Lett. 240B (1990) 333;

G. Parisi, Phys. Lett. 238B (1990) 209.

[3] I. Klebanov, "String Theory in Two Dimensions", Preprint PUPT-1271, July 1991;

D. Kutasov, Some properties of (non)critical strings, Lectures given at ICTP Spring School on String Theory and Quantum Gravity, Trieste, Italy 1991.

[4] S. Das and A. Jevicki, Mod. Phys. Lett. A5 (1990) 1639.

[5] R. Dijkgraaf, G. Moore and R. Plesser, The partition function of 2D string theory, IASSNS-HEP-92/48, YCTP-P22-92.

[6] F. David, Mod. Phys. Lett. A3 (1988) 1651;

J. Distler and H. Kawai, Nucl. Phys. B321 (1989) 509.

[7] J. Polchinski, Nucl. Phys. B 362 (1991) 125;

P. DiFrancesco and D. Kutasov, Phys. Lett. 261B (1991) 385

[8] M. Bershadsky and I. Klebanov, Phys. Rev. Lett. 65 (1990) 3088.

[9] B. Lian and G. Zuckerman, Phys. Lett. 266B (1991) 21;

S. Mukherji, S. Mukhi and A. Sen, Phys. Lett. 266B (1991) 337;

P. Bouwknegt, J. McCarthy and K. Pilch, Comm. Math. Phys. 145 (1992) 541.

[10] E. Witten, Nucl. Phys. B373 (1992) 187;

E. Witten and B. Zwiebach, Nucl. Phys. B377 (1992) 55.

[11] I. Klebanov and A. Polyakov, Mod. Phys. Lett. A6 (1991) 3273.

[12] I. Klebanov, Mod. Phys. Lett. A7 (1992) 723.

[13] E. Witten, Phys. Rev. D44 (1991) 314.

[14] S. Das, Preprint TIFR/TH/92-62, October 1992;

A. Dhar, G. Mandal and S. Wadia, Preprint TIFR/TH/92-63, October 1992.

[15] J. Distler and C. Vafa, Mod. Phys. Lett. A6 (1991) 259.

[16] R.C. Penner, J. Diff. Geom. 27 (1988) 35.

[17] E. Witten, Nucl. Phys. B371 (1992) 191.

[18] B. Gato-Rivera and A. Semikhatov, Phys. Lett. 288B (1992) 38.

[19] M. Bershadsky, W. Lerche, D. Nemeschansky and N. Warner, A BRST operator for non-critical W-Strings, HUTP-A034/92.

[20] V. Knizhnik, A. Polyakov and A.B. Zamolodchikov, Mod. Phys. Lett. A3 (1988) 819.

[21] M. Bershadsky, V. Sadov and C. Vafa, work in progress.

[22] R. Dijkgraaf, E. Verlinde and H. Verlinde, Notes on Topological String Theory and 2D Quantum Gravity, Preprint PUPT-1217, IASSNS-HEP-90/80 (November 1990).

[23] E. Witten, Nucl. Phys. B340 (1990) 281. 
[24] M. Kato and S. Matsuda, in "Conformal Field Theory and Solvable Lattice Models", Adv. Studies in Pure Math. 16, Ed. M. Jimbo, T. Miwa and A. Tsuchiya (Kinokuniya, 1988).

[25] D. Ghoshal, D. Jatkar and S. Mukhi, work in progress.

[26] Y. Kazama and H. Suzuki, Nucl. Phys. B321 (1989) 232.

[27] S. Cecotti and C. Vafa, Nucl. Phys. B367 (1991) 359.

[28] W. Lerche, C. Vafa and, N. Warner, Nucl. Phys. B324 (1989) 427.

[29] K. Gawedzki and A. Kupiainen, Nucl. Phys. B320 (1989) 625.

[30] E. Frenkel, Phys. Lett. B 286 (1992) 71.

[31] G. Moore and N. Seiberg, Phys. Lett. B220 (1989) 422.

[32] A. Gerasimov, A. Morozov, M. Olshanetsky and A. Marshakov, Int. J. Mod. Phys. A5 (1990) 2495.

[33] A.V. Marshakov, Phys. Lett. B224 (1989) 141;

Vl.S. Dotsenko, Nucl. Phys. B338 (1990) 747.

[34] D. Friedan, E. Martinec and S. Shenker, Nucl. Phys. B271 (1986) 93.

[35] P. Horava, Nucl. Phys. B386 (1992) 383.

[36] S. Chaudhuri, H. Dykstra and J. Lykken, Mod. Phys. Lett. A6 (1991) 1665.

[37] C. Itzykson and J. Zuber, Comm. Math. Phys. 134 (1990) 197;

N. Chair and S. Panda, Phys. Lett. 272B (1991) 230;

N. Chair, Rev. Math. Phys. 3 (1991) 285;

C.I. Tan, Mod. Phys. Lett. A6 (1991) 1373; Phys. Rev. D45 (1992) 2862;

M. Srednicki, Mod. Phys. Lett.A7 (1992) 2857;

R. Brower, N. Deo, S. Jain and C.I. Tan, Harvard preprint, HUTP-92/A035.

[38] A. Alekseev and S. Shatashvili, Nucl. Phys. B323 (1989) 719.

[39] N. Marcus and Y. Oz, Preprint TAUP-1962-92, June 1992

[40] V. Sadov, On the spectra of $s l(N) / s l(N)$-cosets and $W_{N}$ gravities, Harvard preprint HUTP-92/A055 .

[41] G. Moore, R. Plesser and S. Ramgoolam, Nucl. Phys. B377 (1992) 143.

[42] R. Dijkgraaf, E. Verlinde and H. Verlinde, Nucl. Phys. B 371 (1992) 269;

M. Bershadsky and D. Kutasov, Phys. Lett. B266 (1991) 345;

J. Distler and P. Nelson, Nucl. Phys. B 374 (1992) 123;

T. Eguchi, H. Kanno and S.-K. Yang, $W_{\infty}$ Algebra in two-dimensional black hole, Preprint NI92004;

H. Ishikawa and M. Kato, Equivalence of BRST cohomologies for 2-d black hole and $c=1$ Liouville theory, preprint, UT-Komaba/92-11.

[43] I. Bars, String propagation on black holes, preprint USC-91-HEP-B3;

Nucl. Phys. B 334 (1990) 125.

[44] T. Eguchi, Mod. Phys. Lett. A7 (1992) 85.

[45] I. Frenkel, H. Garland and G. Zuckerman, Proc. Nat. Acad. Sci. USA 83 (1986) 8442. 
[46] L. Dixon, J. Harvey, C. Vafa and E. Witten, Nucl. Phys. B274 (1986) 285.

[47] S. Cecotti and C. Vafa, Mod. Phys. Lett. A7 (1992) 1715.

[48] E. Zaslow, Topological orbifold models and quantum cohomology rings, preprint, HUTP-92/A065.

[49] S. Hamidi and C. Vafa, Nucl. Phys. B279 (1987) 465;

L. Dixon, D. Friedan, E. Martinec and S. Shenker, Nucl. Phys. B282 (1987) 13.

[50] K. Li, Nucl. Phys. B354 (1991) 711.

[51] M. Bershadsky and E. Frenkel, unpublished;

O. Aharony, O. Ganor, N. Sochen, J. Sonnenschein and S. Yankielowicz, Physical states in $G / G$ models and 2d gravity, Preprint TAUP-1961-92;

O. Aharony, J. Sonnenschein and S. Yankielowicz, $G / G$ models and $W_{N}$ Strings, Preprint TAUP-1977-92;

H.L. Hu and M.Yu, On BRST cohomology of $S L(2, R) / S L(2, R)$ gauged WZNW models, Preprint AS-ITP-92-32;

P. Bouwkneght,J. McCarthy and K. Pilch, Semi-infinite cohomology in CFT and 2d gravity, Preprint CERN-TH. 6646/92.

[52] M. Bershadsky and H. Ooguri, Comm. Math. Phys. 126 (1989) 49.

[53] B.Feigin, E.Frenkel, Comm. Math. Phys. 128 (1990) 161

[54] B.Feigin, E.Frenkel, in Physics and Mathematics of Strings, V.Knizhnik Memorial Volume, eds. L.Brink, e.a., 271-316, World Scientific, 1990

[55] D.Bernard, G.Felder, Comm. Math. Phys. 127 (1990) 145 